\title{
Total Synthesis of Anachelin H
}

\author{
Karl Gademann and Yann Bethuel \\ Laboratorium für Organische Chemie der ETH Zürich \\ $\mathrm{CH}-8093$ Zürich
}

Supporting Information 
Abbreviations. $\mathrm{CDI}=$ Carbonyldiimidazole; $\mathrm{DMAP}=N, N$-Dimethylaminopyridine; EDC $=$ Ethyl-(N, N-dimethylaminopropyl)carbodiimide; ether refers to diethylether; $\mathrm{RT}=$ room temperature; TBS = tert-Butyl-dimethylsilyl; $Z$ = Benzyloxycarbonyl.

General.Unless otherwise stated, chemicals were purchased from Fluka, ABCR or Acros and used without further purification. $\mathrm{H}-\mathrm{Ser}(\mathrm{OBn})-\mathrm{OH}$ was purchased from Senn Chemicals, Switzerland. Commercially available TBSOTf was distilled and stored at 4 ${ }^{\circ} \mathrm{C} .2,6$-Lutidine was distilled from $\mathrm{CaH}_{2}$ and stored at $4{ }^{\circ} \mathrm{C}$. CDI was recrystallized from THF. Solvents for work-up and chromatography were distilled from technical quality. Solvents used for chemical transformations were either puriss quality or dried over columns of dried aluminium oxide. Z-Ser(OBn)-OH was prepared according to reference [1]. 2-O-Bn-salicylic acid was prepared according to reference [2]. (S)-[2(3,4-Dihydroxy-phenyl)-1-dimethylcarbamoyl-ethyl]-carbamic acid tert-butyl ester was prepared according to [3]. 7-Benzyloxy-(6S)-6-benzyloxycarbonylamino-(5R)-5hydroxy-3-oxo-heptanoic acid methyl ester was prepared according to [3]. $\mathrm{MgCl}_{2}$ was stored and weighed in a glove box under inert atmosphere.

Reactions were run under an atmosphere of Ar in dry glassware (at least $24 \mathrm{~h}$ in an oven at $140{ }^{\circ} \mathrm{C}$, followed by heating with a heat gun under high vacuum). Analytical thin layer chromatograpy (TLC) was performed on Merck silica gel 60 F254 plates (0.25 $\mathrm{mm}$ thickness) precoated with a fluorescent indicator. The developed plates were examined under UV light and stained with ceric ammonium phosphate (CAM) stain or $\mathrm{KMnO}_{4}$ stain followed by heating. Flash chromatography was performed using silica gel 60 (230-400 mesh) from Fluka. All ${ }^{1} \mathrm{H}$ and ${ }^{13} \mathrm{C}$ NMR spectra were recorded using either Varian Gemini $\left(300 \mathrm{MHz}\left({ }^{1} \mathrm{H}\right)\right.$ or $\left.75 \mathrm{MHz}\left({ }^{13} \mathrm{C}\right)\right)$, Varian Mercury $\left(300 \mathrm{MHz}\left({ }^{1} \mathrm{H}\right)\right.$ or $\left.75 \mathrm{MHz}\left({ }^{13} \mathrm{C}\right)\right)$, Bruker AMX $\left(500 \mathrm{MHz}\left({ }^{1} \mathrm{H}\right)\right.$ or $\left.125 \mathrm{MHz}\left({ }^{13} \mathrm{C}\right)\right)$ or Bruker DMX $500 \mathrm{MHz}$ 
$\left({ }^{1} \mathrm{H}\right)$ or $\left.125 \mathrm{MHz}\left({ }^{13} \mathrm{C}\right)\right)$ FT spectrometers at ambient temperature, chemical shifts $\delta$ are given in ppm, coupling constants $J$ are in $\mathrm{Hz}$. IR spectra were recorded as $\mathrm{CHCl}_{3}$ solution using a Perkin Elmer RX I FT-IR spectrometer, absorptions are given in $\mathrm{cm}^{-1}$. Optical rotations were measured using a $1 \mathrm{~mL}$ cell with a $1 \mathrm{dm}$ path length on a Jasco DIP 1000 digital polarimeter, the concentration $c$ is given in $\mathrm{g} / 100 \mathrm{~mL}$. Elemental analyses were performed by the Mikroanalyse Labor of the Laboratorium für Organische Chemie der ETH Zürich. All mass spectra were recorded by the Masspectroscopy Service of the Laboratorium für Organische Chemie der ETH Zürich on a lon spec Ultima 4.7 spectrometer in 2,5-DHB matrix using MALDI, and on TSQ 7000 using ESI. Fragment ions are given in $\mathrm{m} / \mathrm{z}$ with relative intensities (\%) in parentheses. 

tert-butyl ester<smiles>CN(C)C(=O)[C@H](Cc1ccc(O)c(O)c1)NC(=O)[O-]</smiles><smiles>CN(C)C(=O)[C@H](Cc1ccc(OCc2ccccc2)c(OCc2ccccc2)c1)NC(=O)c1ccccc1</smiles>

(S)-[2-(3,4-Dihydroxy-phenyl)-1-dimethylcarbamoyl-ethyl]-carbamic acid tert-butyl ester $(6.48 \mathrm{~g}, 20 \mathrm{mmol})$ was dissolved in acetone $(150 \mathrm{~mL})$ and $\mathrm{Cs}_{2} \mathrm{CO}_{3}(19.55 \mathrm{~g}, 60$ mmol, $3 \mathrm{eq}$ ) was added. The reaction mixture was stirred at this temperature for 15 minutes and the color turned red. Then benzyl bromide $(7.13 \mathrm{~mL}, 60 \mathrm{mmol}, 3 \mathrm{eq})$ was added and the reaction mixture was refluxed for 4 hours. The solvent was evaporated under reduced pressure and the residue taken up in AcOEt. It was washed $3 x$ with citric acid, $3 x$ with satd. $\mathrm{NaHCO}_{3}$ soln., $2 x$ with $\mathrm{H}_{2} \mathrm{O}$ and $2 x$ with brine. The organic phase was dried over $\mathrm{MgSO}_{4}$, filtered and evaporated under reduced pressure. The obtained solid was recrystallised from AcOEt/hexane 1:5 to give the title compound. FC (Hexane / AcOEt 1:1) of the evaporated mother liquor gave another $2 \mathrm{~g}$ to finally yield 15 (9.6 g, $19 \mathrm{mmol}, 95 \%)$. White solid. $\mathrm{M}_{\mathrm{p}}=85-88{ }^{\circ} \mathrm{C} . \mathrm{R}_{\mathrm{f}}=0.3$ (AcOEt/Hexane 1:1). $[\alpha]_{D}=+27.5\left(\mathrm{C}=1.05, \mathrm{CHCl}_{3}, \mathrm{~T}=28.5^{\circ} \mathrm{C}\right) .{ }^{1} \mathrm{H}-\mathrm{NMR}\left(\mathrm{CDCl}_{3}, 300 \mathrm{MHz}\right) \delta 1.42(\mathrm{~s}$, $9 \mathrm{H}), 2.46(\mathrm{~s}, 3 \mathrm{H}), 2.76(\mathrm{~s}, 3 \mathrm{H}), 2.81\left(\mathrm{dd}, 1 \mathrm{H}, J_{1}=9.33, J_{2}=13.07\right), 2.91\left(\mathrm{dd}, 1 \mathrm{H}, J_{1}=\right.$ 5.6, $\left.J_{2}=13.07\right), 4.69-4.79(\mathrm{~m}, 1 \mathrm{H}), 5.11(\mathrm{~s}, 4 \mathrm{H}), 5.52(\mathrm{~d}, 1 \mathrm{H}, \mathrm{J}=8.4), 6.68\left(\mathrm{dd}, 1 \mathrm{H}, J_{1}=\right.$ 1.56, $\left.\mathrm{J}_{2}=8.41\right), 6.82(\mathrm{~m}, 2 \mathrm{H}), 7.24-7.48(\mathrm{~m}, 10 \mathrm{H}) .{ }^{13} \mathrm{C}-\mathrm{NMR}\left(\mathrm{CDCl}_{3}, 75 \mathrm{MHz}\right) 28.5,35.5$, $36.8,40.0,51.4,71.2,71.3,76.8,79.5,115.2,116.0,122.2,127.3,127.69,127.75$, 128.3, 128.4, 129.9, 137.1, 137.2, 147.6, 148.7, 155.0, 171.4. IR 3293w, 1704s, 1641s, 1509s. MS $527\left(100,[\mathrm{M}+\mathrm{Na}]^{+}\right)$. 

tert-butyl ester<smiles>CN(C)C(=O)N[C@@H](Cc1ccc(OCc2ccccc2)c(OCc2ccccc2)c1)C(=O)O</smiles><smiles>CN(C)CC(Cc1ccc(OCc2ccccc2)c(OCc2ccccc2)c1)NC(=O)c1ccccc1</smiles>

(2S)-[2-(3,4-Bis-benzyloxy-phenyl)-1-dimethylcarbamoyl-ethyl]-carbamic acid tert-butyl ester $(7.6 \mathrm{~g}, 14.8 \mathrm{mmol})$ was dissolved in $\mathrm{CH}_{2} \mathrm{Cl}_{2}(60 \mathrm{~mL})$ and cooled to $0{ }^{\circ} \mathrm{C}$. Then TFA $(60 \mathrm{~mL})$ was added and the reaction mixture was stirred for 1 hour at this temperature and for 1 hour at RT. It was then neutralized by the addition of $\mathrm{NaOH}(1 \mathrm{M})$ and extracted 3x with AcOEt. The combined organic phases were dried over $\mathrm{MgSO}_{4}$, filtered and evaporated under reduced pressure. It was directly used without further purification in the next step.

The deprotected amine was dissolved in THF $(40 \mathrm{~mL})$ and cooled to $0{ }^{\circ} \mathrm{C}$. Then $\mathrm{BH}_{3} \cdot \mathrm{THF}(1 \mathrm{M})(44.4 \mathrm{~mL}, 44.4 \mathrm{mmol}, 3 \mathrm{eq})$ was added dropwise and the reaction mixture was refluxed over night. It was cooled to $0^{\circ} \mathrm{C}$ and $\mathrm{HCl}(12 \mathrm{~N})$ was added until $\mathrm{pH}$ reached 2-3. THF was evaporated under reduced pressure and $\mathrm{NaOH}(6 \mathrm{~N})$ was added until $\mathrm{pH}=14$. It was extracted $3 \mathrm{x}$ with $\mathrm{AcOEt}$ and the combined organic phases were dried over $\mathrm{MgSO}_{4}$, filtered and evaporated under reduced pressure. It was used in the next step without further purification.

The diamine was dissolved in a mixture of dioxane/water $20 \mathrm{~mL}: 20 \mathrm{~mL}$ and $\mathrm{NaOH}$ (1M, $15.54 \mathrm{mmol}, 15.54 \mathrm{~mL}, 1.05 \mathrm{eq})$ was added. $\mathrm{Boc}_{2} \mathrm{O}(3.88 \mathrm{~g}, 17.76 \mathrm{mmol}, 1.2 \mathrm{eq})$ were added and the reaction mixture was stirred at RT over night. Dioxane was removed and the residual water extracted with $3 x$ AcOEt. The combined organic phases were dried over $\mathrm{MgSO}_{4}$, filtered and evaporated under reduced pressure. FC (AcOEt/Hexane 8:2) gave 16 (3.8 g, $7.8 \mathrm{mmol}, 53 \%)$. Colorless oil. $\mathrm{R}_{\mathrm{f}}=0.4\left(\mathrm{CH}_{2} \mathrm{Cl}_{2} /\right.$ 
$\mathrm{MeOH} 9: 1) \cdot[\alpha]_{\mathrm{D}}=+19.95\left(\mathrm{C}=2.05, \mathrm{CHCl}_{3}, \mathrm{~T}=27.1^{\circ} \mathrm{C}\right) .{ }^{1} \mathrm{H}-\mathrm{NMR}\left(\mathrm{CDCl}_{3}, 300 \mathrm{MHz}\right) \delta$ $1.44(\mathrm{~s}, 9 \mathrm{H}), 2.06-2.16(\mathrm{~m}, 2 \mathrm{H}), 2.18(\mathrm{~s}, 6 \mathrm{H}), 2.71\left(\mathrm{dd}, 1 \mathrm{H}, J_{1}=6.54, J_{2}=13.7\right), 2.84(\mathrm{dd}$, $\left.1 \mathrm{H}, J_{1}=4.05, J_{2}=13.7\right), 3.78-3.81(\mathrm{~m}, 1 \mathrm{H}), 4.61-4.69(\mathrm{~m}, 1 \mathrm{H}), 5.13(\mathrm{~s}, 2 \mathrm{H}), 5.15(\mathrm{~s}, 2 \mathrm{H})$, $6.69\left(\mathrm{dd}, 1 \mathrm{H}, J_{1}=1.86, J_{2}=8.09\right), 6.82(\mathrm{~d}, 1 \mathrm{H}, J=1.86), 6.87(\mathrm{~d}, 1 \mathrm{H}, J=8.4), 7.23-7.5$ (m, 10H). ${ }^{13} \mathrm{C}-\mathrm{NMR}\left(\mathrm{CDCl}_{3}, 75 \mathrm{MHz}\right) 28.5,38.4,45.6,61.7,71.3,71.4,79.1,115,116.6$, 122.5, 127.2, 127.3, 127.6, 127.7, 128.3, 131.1, 137.2, 137.3, 147.4, 148.5, 155.6. IR $3365 w, 2971 w, 2768 w, 1682 s, 1523 s . M S 391.2\left(48,[M-B o c]^{+}\right)$. HRMS calcd. for $\mathrm{C}_{25} \mathrm{H}_{31} \mathrm{~N}_{2} \mathrm{O}_{2}$ (M-Boc) $)^{+}$391.2380, found: 391.2377.

\section{(3S)-6,7-Bis-benzyloxy-3-tert-butoxycarbonylamino-1,1-dimethyl-1,2,3,4-}

tetrahydro-quinolinium; chloride<smiles>CN(C)CC(Cc1ccc(OCc2ccccc2)c(OCc2ccccc2)c1)NC(=O)OCc1ccccc1</smiles><smiles>C[N+]1(C)C[C@H](NC(=O)c2ccccc2)Cc2cc(OCc3ccccc3)c(OCc3ccccc3)cc21</smiles>

(2S)-[2-(3,4-Bis-benzyloxy-phenyl)-1-dimethylaminomethyl-ethyl]-carbamic acid tertbutyl ester (1.6 g, $3.26 \mathrm{mmol})$ was dissolved in $\mathrm{MeOH}(30 \mathrm{~mL})$, and $\mathrm{AcOH}(300 \mu \mathrm{l})$. The reaction mixture was flushed $3 x$ with argon and $\mathrm{Pd} / \mathrm{C}(10 \%, 350 \mathrm{mg})$ was added. Then it was flushed with $\mathrm{H}_{2}$ and stirred at RT for 4 hours. $\mathrm{Pd} / \mathrm{C}$ was filtered over Celite and the solvent evaporated under reduced pressure.

17 was dissolved in $\mathrm{CH}_{2} \mathrm{Cl}_{2}(33 \mathrm{~mL})$ and dianisyltelluriumoxide (1.28 g, $3.59 \mathrm{mmol}, 1.1$ eq) was added. After 20 minutes the color turned red, and the reaction mixture was stirred for another 3 hours at RT. The organic phase was extracted $3 x$ with water and the water phases washed $5 x$ with $\mathrm{CH}_{2} \mathrm{Cl}_{2}$. Lyophilization gave 18 that was directly introduced in the next step. 
The compound was dissolved in dry aceton $(30 \mathrm{~mL})$ and $\mathrm{Cs}_{2} \mathrm{CO}_{3}(3.19 \mathrm{~g}, 9.78 \mathrm{mmol}, 3$ eq) was added. After addition of $\mathrm{BnBr}(3.27 \mathrm{~mL}, 9.78 \mathrm{mmol}, 3 \mathrm{eq})$, the reaction mixture was refluxed for 4 hours and the solvent then evaporated under reduced pressure. The residue was taken up in AcOEt and washed $3 x$ with $\mathrm{H}_{2} \mathrm{O}$. The organic phase was dried over $\mathrm{Na}_{2} \mathrm{SO}_{4}$, filtered and evaporated under reduced pressure. $\mathrm{FC}\left(\mathrm{CH}_{2} \mathrm{Cl}_{2} / \mathrm{MeOH} 9: 1\right)$ gave 19 (630 mg, $1.3 \mathrm{mmol}, 40 \%)$. Off-white solid. $\mathrm{R}_{\mathrm{f}}=0.5\left(\mathrm{CH}_{2} \mathrm{Cl}_{2} / \mathrm{MeOH} 9: 1\right) \cdot[\alpha]_{\mathrm{D}}=$ $+8.16\left(\mathrm{C}=1, \mathrm{CHCl}_{3}, \mathrm{~T}=25.6{ }^{\circ} \mathrm{C}\right) .{ }^{1} \mathrm{H}-\mathrm{NMR}\left(\mathrm{CDCl}_{3}, 300 \mathrm{MHz}\right) \delta 1.39(\mathrm{~s}, 9 \mathrm{H}), 2.1-2.16$ (m, 2H), 3.0 (br. d, 1H), 3.15 (br. dd, 1H), $3.73(\mathrm{~s}, 3 \mathrm{H}), 3.95-4.05(\mathrm{~m}, 1 \mathrm{H}), 4.1-4.3(\mathrm{~m}$, 2H), 5.1 (s, 2H), 5.35 (s, 2H), 6.6 (br. s, 2H), 7.25-7.38 (m, 7H), 7.49 (d, 1H, J = 7.16), 7.61 (s, 1H). ${ }^{13} \mathrm{C}-\mathrm{NMR}\left(\mathrm{CDCl}_{3}, 75 \mathrm{MHz}\right) 28.4,33.1,41.2,58.7,66.4,71.0,72.1,107.8$, 114.5, 127.0, 127.7, 127.9, 128.1, 128.4, 128.6, 135.9, 136.3, 148.5, 149.9, 194.1. IR 3686-3107w, 1700m, 1518m. MS $433.2\left(100,[\mathrm{M}-\mathrm{tBu}]^{+}\right), 489.3\left(46, \mathrm{M}^{+}\right)$. HRMS calcd. for $\mathrm{C}_{30} \mathrm{H}_{37} \mathrm{~N}_{2} \mathrm{O}_{4}(\mathrm{M})^{+}:$: 489.2748, found: 489.2742 .

\section{Boc-L-Thr(OBn)-D-Ser(OBn)-L-Ser(OBn)-(3S)-THQ(OBn); chloride}<smiles>CC(C)(C)OC(=O)NC1Cc2cc(OCc3ccccc3)c(OCc3ccccc3)cc2[N+](C)(Cl)C1</smiles>

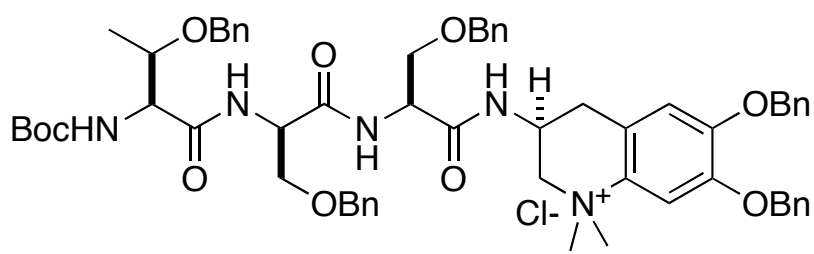

(3S)-6,7-Bis-benzyloxy-3-tert-butoxycarbonylamino-1,1-dimethyl-1,2,3,4-tetrahydroquinolinium chloride $(50 \mathrm{mg}, 95.3 \mu \mathrm{mol})$ was dissolved in dioxane $(1 \mathrm{~mL})$, cooled to 0 ${ }^{\circ} \mathrm{C}$ and $\mathrm{HCl}$.dioxane $(4 \mathrm{M})(0.48 \mathrm{mmol}, 480 \mu \mathrm{l}, 5 \mathrm{eq})$ was added. The reaction mixture was stirred at $0{ }^{\circ} \mathrm{C}$ for 1 hour and 3 hours at RT. Then the solvent was removed under reduced pressure and the product dried under high vacuum overnight. 
Boc-L-Ser(OBn) (1.2 eq) was dissolved in DMF (1 mL) with iBuOCOCl (15 $\mu \mathrm{l}, 150 \mu \mathrm{mol}$ $1.2 \mathrm{eq})$ and $\operatorname{NMM}(21 \mu \mathrm{l}, 191 \mu \mathrm{mol}, 2 \mathrm{eq})$ and cooled to $-18^{\circ} \mathrm{C}$. The reaction mixture was stirred for 30 min and the amine salt dissolved in DMF $(0.5 \mathrm{~mL}), \mathrm{NMM}(21 \mu \mathrm{l}, 191$ umol, 2 eq) was added dropwise. It was allowed to reach RT gradually overnight. Then the solvent was evaporated under reduced pressure and the residue taken up in AcOEt. It was washed 3xwith $\mathrm{H}_{2} \mathrm{O}$ and the combined water phases $3 x$ extracted with AcOEt. The combined organic phases were dried over $\mathrm{Na}_{2} \mathrm{SO}_{4}$, filtered and evaporated under reduced pressure. $\mathrm{FC}\left(\mathrm{CH}_{2} \mathrm{Cl}_{2} / \mathrm{MeOH} 90: 10\right)$ gave the expected product (47.4 $\mathrm{mg}, 67.7 \mu \mathrm{mol}, 71 \%)$.

It was dissolved in dioxane $(1 \mathrm{~mL})$, cooled to $0{ }^{\circ} \mathrm{C}$ and $\mathrm{HCl}$.dioxane $(4 \mathrm{M}, 85 \mu \mathrm{l}$, $338 \mu \mathrm{mol}, 5 \mathrm{eq})$ was added. The reaction mixture was stirred for 1 hour at $0{ }^{\circ} \mathrm{C}$ and 3 hours at RT. Then the solvent was evaporated under reduced pressure and the product dried under high vacuum overnight.

Boc-L-Thr(OBn)-D-Ser(OBn) (40 mg, $81.2 \mu \mathrm{mol}, 1.2 \mathrm{eq})$ was dissolved in DMF (1 mL) with iBuOCOCl (10.6 $\mu \mathrm{l}, 81.2 \mu \mathrm{mol}, 1.2 \mathrm{eq})$ and NMM (15 $\mu \mathrm{l}, 135 \mu \mathrm{mol}, 2$ eq) and cooled to $-18{ }^{\circ} \mathrm{C}$. The reaction mixture was stirred for $30 \mathrm{~min}$ and the amine salt dissolved in DMF (0.5 mL), NMM (15 $\mu \mathrm{l}, 135 \mu \mathrm{mol}, 2 \mathrm{eq})$ was added dropwise. It was allowed to reach RT gradually over night. Then the solvent was evaporated under reduced pressure and the residue taken up in AcOEt. It was washed 3 times with $\mathrm{H}_{2} \mathrm{O}$ and the combined water phases $3 x$ extracted with AcOEt. The combined organic phases were dried over $\mathrm{Na}_{2} \mathrm{SO}_{4}$, filtered and evaporated under reduced pressure. $\mathrm{FC}$ $\left(\mathrm{CH}_{2} \mathrm{Cl}_{2} / \mathrm{MeOH} 90: 10\right)$ gave the title compound (34 mg, $\left.33.1 \mu \mathrm{mol}, 49 \%\right)$. Colorless oil. $\mathrm{R}_{\mathrm{f}}=0.33\left(\mathrm{CH}_{2} \mathrm{Cl}_{2} / \mathrm{MeOH} 90: 10\right) \cdot[\alpha]_{\mathrm{D}}=+4.36\left(\mathrm{C}=1.825, \mathrm{CHCl}_{3}, \mathrm{~T}=25.1{ }^{\circ} \mathrm{C}\right) .{ }^{1} \mathrm{H}-\mathrm{NMR}$ $\left(\mathrm{CDCl}_{3}, 300 \mathrm{MHz}\right) \delta 1.19(\mathrm{~d}, 3 \mathrm{H}, \mathrm{J}=6.23), 1.33(\mathrm{~s}, 9 \mathrm{H})$, 2.80-2.90 (m, 2H), 3.15-3.3 (m, 
$1 \mathrm{H}), 3.45(\mathrm{~s}, 6 \mathrm{H}), 3.6-3.75(\mathrm{~m}, 4 \mathrm{H}), 3.9-4.0(\mathrm{~m}, 2 \mathrm{H}), 4.05-4.2(\mathrm{~m}, 1 \mathrm{H}), 4.3-4.45(\mathrm{~m}, 1 \mathrm{H})$, $4.43(\mathrm{~s}, 2 \mathrm{H}), 4.47(\mathrm{~s}, 2 \mathrm{H}), 4.48(\mathrm{~s}, 2 \mathrm{H}), 4.6-4.7(\mathrm{~m}, 1 \mathrm{H}), 4.75-4.82(\mathrm{~m}, 1 \mathrm{H}), 5.1(\mathrm{~d}, 2 \mathrm{H}, \mathrm{J}=$ 3.11), $5.31(\mathrm{~s}, 2 \mathrm{H}), 5.63(\mathrm{~d}, 1 \mathrm{H}, J=7.47), 6.55(\mathrm{~s}, 1 \mathrm{H}), 7.02-7.41(\mathrm{~m}, 25 \mathrm{H}), 7.82(\mathrm{~d}, 1 \mathrm{H}, J$ $=7.78), 8.51(\mathrm{~d}, 1 \mathrm{H}, J=5.92), 8.68(\mathrm{~d}, 1 \mathrm{H}, J=6.53)$. IR $3317 w, 1705 w, 1664 m, 1518 m$. MS $1034.5\left(100,[M]^{+}\right)$. HRMS calcd. for $\mathrm{C}_{61} \mathrm{H}_{72} \mathrm{~N}_{5} \mathrm{O}_{10}(\mathrm{M})^{+}:$1034.5274, found: 1034.5264.

\section{(2,2-dimethyl-6-methylene-6H-[1,3]dioxin-4-yloxy)-trimethyl-silane}<smiles>CC1=CC(=O)OC(C)(C)O1</smiles><smiles>C=C1C=C(O[Si](C)(C)C)OC(C)(C)O1</smiles>

As a slight modification of a literature procedure [4], to a solution of $i \mathrm{Pr}_{2} \mathrm{NH}(1.29 \mathrm{~mL}$, $9.18 \mathrm{mmol}, 1.2 \mathrm{eq})$ in anhydrous $\mathrm{THF}(5 \mathrm{~mL})$ at $0{ }^{\circ} \mathrm{C}$ was added $n$ BuLi $(1.32 \mathrm{M}$ in hexane, $5.21 \mathrm{~mL}, 9.18 \mathrm{mmol}, 1.2 \mathrm{eq})$ over 15 minutes. The clear solution was stirred at $0{ }^{\circ} \mathrm{C}$ for $20 \mathrm{~min}$ and then cooled to $-78^{\circ} \mathrm{C} .2,2,6$-Trimethyl-[1,3]dioxinone $(1 \mathrm{~mL}, 7.65$ mmol) was added neat over 10 minutes and the light yellow solution was stirred at -78 ${ }^{\circ} \mathrm{C}$ for 1 hour. TMSCI (1.36 mL, $\left.10.7 \mathrm{mmol}, 1.4 \mathrm{eq}\right)$ was added dropwise over $15 \mathrm{~min}$ and the reaction mixture was stirred for an additional $30 \mathrm{~min}$ at $-78{ }^{\circ} \mathrm{C}$. The suspension was then allowed to warm to ambient temperature over $90 \mathrm{~min}$. It was filtered over anhydrous $\mathrm{Na}_{2} \mathrm{SO}_{4}$ under argon. The orange filtrate was concentrated under reduced pressure and the remaining oil distilled under reduced pressure $\left(0.5 \mathrm{mbar}, \mathrm{T}<50{ }^{\circ} \mathrm{C}\right)$ to give (2,2-dimethyl-6-methylene-6H-[1,3]dioxin-4-yloxy)-trimethyl-silane (1.31 g, 6.1 mmol, 80\%). Colorless oil. ${ }^{1} \mathrm{H}-\mathrm{NMR}\left(\mathrm{CDCl}_{3}, 300 \mathrm{MHz}\right) \delta 0.27$ (s, 9H), 1.55 (s, 6H), 3.88 (s, 1H), $4.07(\mathrm{~s}, 1 \mathrm{H}), 4.65(\mathrm{~s}, 1 \mathrm{H})$. 


\section{[1-(tert-Butyl-dimethyl-silanyloxymethyl)-3-(2,2-dimethyl-6-oxo-6H-[1,3]dioxin-4- yl)-((3S)-2-hydroxy-propyl]-(2S)carbamic acid benzyl ester}<smiles></smiles>

Following a modified literature procedure [5], a solution of [1-(tert-Butyl-dimethylsilanyloxymethyl)-(2S)-2-oxo-ethyl]-carbamic acid benzyl ester (298 mg, $0.89 \mathrm{mmol}$ ) in dry $\mathrm{CH}_{2} \mathrm{Cl}_{2}(1 \mathrm{~mL})$ was cooled to $0^{\circ} \mathrm{C}$ and $\mathrm{Eu}(\mathrm{fod})_{3}(185 \mathrm{mg}, 0.178 \mathrm{mmol}, 0.2 \mathrm{eq})$ was added. After 10 minutes (2,2-dimethyl-6-methylene-6H-[1,3]dioxin-4-yloxy)-trimethylsilane (190 mg, $0.89 \mathrm{mmol}, 1 \mathrm{eq})$ dissolved in $\mathrm{CH}_{2} \mathrm{Cl}_{2}(1 \mathrm{~mL})$ was added dropwise. The reaction mixture was then stirred overnight reaching $\mathrm{RT}$ gradually. $\mathrm{CH}_{2} \mathrm{Cl}_{2}$ was removed under reduced pressure and the residue was taken up with AcOEt. It was washed $3 x$ with citric acid (10\%) and $3 x$ with satd. $\mathrm{NaHCO}_{3}$. The organic phase was dried over $\mathrm{Na}_{2} \mathrm{SO}_{4}$, filtered and evaporated under reduced pressure. FC (AcOEt/Hexane 3:7) gave the title compound (199 mg, $0.41 \mathrm{mmol}, 47 \%$ ). White solid. $M_{p}=60-63{ }^{\circ} \mathrm{C} . R_{f}=0.21\left(\right.$ AcOEt/Hexane 3:7). $[\alpha]_{D}=-1.07\left(\mathrm{C}=3.2, \mathrm{CHCl}_{3}, \mathrm{~T}=26^{\circ} \mathrm{C}\right)$. ${ }^{1} \mathrm{H}-\mathrm{NMR}\left(\mathrm{CDCl}_{3}, 300 \mathrm{MHz}\right) \delta 0.056(\mathrm{~s}, 3 \mathrm{H}), 0.071(\mathrm{~s}, 3 \mathrm{H}), 0.88(\mathrm{~s}, 9 \mathrm{H}), 2.34\left(\mathrm{dd}, 1 \mathrm{H}, J_{1}=\right.$ 4.67, $\left.J_{2}=14.95\right), 2.44\left(\mathrm{dd}, 1 \mathrm{H}, J_{1}=8.5, J_{2}=14.95\right), 3.33(\mathrm{~s}, 1 \mathrm{H}), 3.63-3.66(\mathrm{~m}, 1 \mathrm{H})$, $3.86(\mathrm{~d}, 2 \mathrm{H}, \mathrm{J}=3.42), 4.26-4.29(\mathrm{~m}, 1 \mathrm{H}), 5.12(\mathrm{~s}, 2 \mathrm{H}), 5.33(\mathrm{~s}, 1 \mathrm{H}), 5.41(\mathrm{br} . \mathrm{d}, 1 \mathrm{H}, J=$ 9.03), 7.32-7.37 (m, 5H). ${ }^{13} \mathrm{C}-\mathrm{NMR}\left(\mathrm{CDCl}_{3}, 75 \mathrm{MHz}\right)-5.5,18.2,24.8,25.4,25.8,38.6$, $54.1,66.0,67.0,69.9,95.3,106.2,128.0,128.2,128.5,136.1,156.2,160.8,168.1$. 

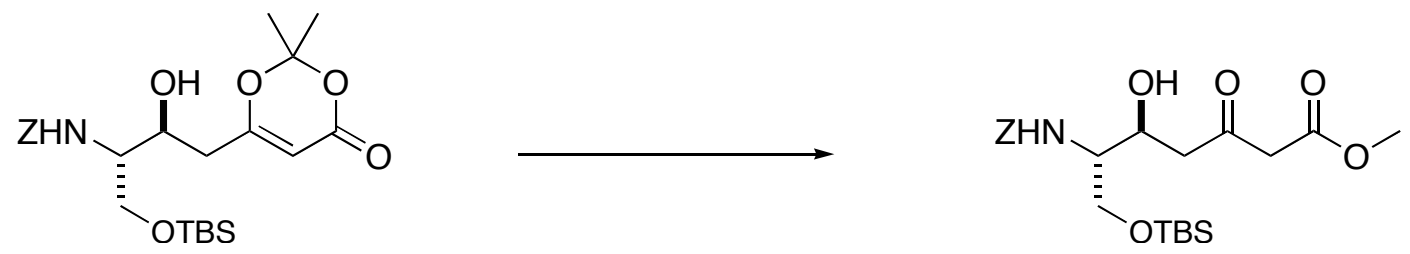

To a solution of [1-(tert-Butyl-dimethyl-silanyloxymethyl)-3-(2,2-dimethyl-6-oxo-6H[1,3]dioxin-4-yl)-((3S)-2-hydroxy-propyl]-(2S)carbamic acid benzyl ester (175 mg, 0.36 $\mathrm{mmol})$ in toluene $(3 \mathrm{~mL})$ was added $\mathrm{MeOH}(58 \mu \mathrm{l}, 1.44 \mathrm{mmol}, 4 \mathrm{eq})$ and the reaction mixture was refluxed for 4 hours. Then it was cooled until RT and the solvents were removed under reduced pressure. FC (AcOEt/Hexane 2.5:7.5) gave the title compound 5 (141 mg, $0.31 \mathrm{mmol}, 85 \%) . \mathrm{R}_{\mathrm{f}}=0.5$ (AcOEt/Hexane 4:6). $[\alpha]_{\mathrm{D}}=+0.65\left(\mathrm{C}=2, \mathrm{CHCl}_{3}\right.$, $\left.\mathrm{T}=27.2^{\circ} \mathrm{C}\right) .{ }^{1} \mathrm{H}-\mathrm{NMR}\left(\mathrm{CDCl}_{3}, 300 \mathrm{MHz}\right) \delta 0.051(\mathrm{~s}, 3 \mathrm{H}), 0.06(\mathrm{~s}, 3 \mathrm{H}), 0.87(\mathrm{~s}, 9 \mathrm{H}), 2.65$ (dd, $\left.1 \mathrm{H}, J_{1}=4.35, J_{2}=17.44\right), 2.79\left(\mathrm{dd}, 1 \mathrm{H}, J_{1}=8.4, J_{2}=17.43\right), 3.34(\mathrm{~s}, 1 \mathrm{H}), 3.47(\mathrm{~s}$, 2H), 3.62-3.63 (m, 1H), $3.72(\mathrm{~s}, 3 \mathrm{H}), 3.8(\mathrm{~d}, 2 \mathrm{H}, \mathrm{J}=4.35), 4.44-4.49(\mathrm{~m}, 1 \mathrm{H}), 5.1(\mathrm{~s}, 2 \mathrm{H})$, 5.32 (br. d, $J=9.34), 7.31-7.37(\mathrm{~m}, 5 \mathrm{H}) .{ }^{13} \mathrm{C}-\mathrm{NMR}\left(\mathrm{CDCl}_{3}, 75 \mathrm{MHz}\right)$-5.4, 18.4, 26.0, $47.0,49.8,52.6,54.5,65.0,67.1,68.1,128.4,128.4,128.8,136.6,156.7,167.5,202.5$. IR 3647-3170m, 1744m, 1716s. MS $476.2\left(100,[\mathrm{M}+\mathrm{Na}]^{+}\right), 444.2\left(79,[\mathrm{M}-\mathrm{MeOH}+\mathrm{Na}]^{+}\right.$. HRMS calcd. for $\mathrm{C}_{22} \mathrm{H}_{35} \mathrm{NO}_{7} \mathrm{SiNa}(\mathrm{M}+\mathrm{Na})^{+}:$476.2075, found: 476.2069 . 
hydroxy-3-oxo-heptanoic acid methyl ester<smiles>[Z]N[C@@H](CO[Sb])[C@H](O)CC(=O)CC(=O)OC</smiles><smiles>[Z]N[C@@H](C[OH2+])[C@H](O)C[C@@H](O)CC(=O)OC</smiles>

A solution of (6S)-6-Benzyloxycarbonylamino-7-(tert-butyl-dimethyl-silanyloxy)-(5S)-5hydroxy-3-oxo-heptanoic acid methyl ester $(80 \mathrm{mg}, 0.18 \mathrm{mmol})$ in $\mathrm{CH}_{3} \mathrm{CN}(0.5 \mathrm{~mL})$ was cooled to $-35{ }^{\circ} \mathrm{C}$ and $\mathrm{Me}_{4} \mathrm{~N}(\mathrm{OAc})_{3} \mathrm{BH}(331 \mathrm{mg}, 1.26 \mathrm{mmol}, 7 \mathrm{eq})$ dissolved in $\mathrm{CH}_{3} \mathrm{CN} / \mathrm{AcOH}(0.5 \mathrm{~mL} / 0.5 \mathrm{~mL})$ was added. The reaction mixture was stirred at this temperature for 62 hours. It was then allowed to reach $0{ }^{\circ} \mathrm{C}$ and a saturated solution of Na-K-tartrate was added. The solution was stirred at this temperature for 4 hours. The phases were separated and the aqueous phase extracted $3 x$ with AcOEt. The combined organic layers were washed with brine, dried over $\mathrm{Na}_{2} \mathrm{SO}_{4}$, filtered and evaporated under reduced pressure. The NMR of this compound showed a pure compound (d.r. = $97: 3$ ) which was directly used in the next step.

(6S)-6-Benzyloxycarbonylamino-(3S,5S)-3,5,7-tris-(tert-butyl-dimethyl-silanyloxy)heptanoic acid methyl ester<smiles>[R15]C[C@H](N[Z])[C@@H](O)C[C@@H](O)CC(=O)OC</smiles>

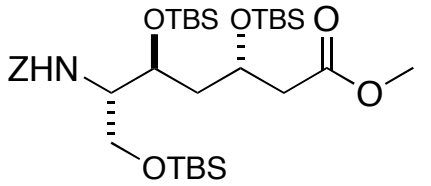

A solution of (6S)-6-Benzyloxycarbonylamino-7-(tert-butyl-dimethyl-silanyloxy)-(3S,5S)4,5-hydroxy-3-oxo-heptanoic acid methyl ester (80.0 mg, $0.18 \mathrm{mmol}$ ) was dissolved in dry $\mathrm{CH}_{2} \mathrm{Cl}_{2}(0.8 \mathrm{~mL})$ and cooled to $-20^{\circ} \mathrm{C} .2,6$ lutidine $(84 \mu \mathrm{l}, 0.72 \mathrm{mmol}, 4 \mathrm{eq})$ was added dropwise followed by the dropwise addition of TBSOTf (124 $\mu$ l, $0.54 \mathrm{mmol}, 3$ eq). The reaction mixture was stirred at $-20{ }^{\circ} \mathrm{C}$ for $1 \mathrm{~h} 30$. It was diluted with $\mathrm{CH}_{2} \mathrm{Cl}_{2}(3$ 
$\mathrm{mL}$ ), washed $3 \mathrm{x}$ with citric acid (10\%) and $2 \mathrm{x}$ with $\mathrm{H}_{2} \mathrm{O}$. It was dried over $\mathrm{MgSO}_{4}$, filtered and evaporated under reduced pressure. FC (AcOEt/hexane 5/95) gave 8 (62.0 $\mathrm{mg}, 0.09 \mathrm{mmol}, 52 \%$ over 2 steps). $R_{\mathrm{f}}=0.53$ (AcOEt/hexane 1:9). $[\alpha]_{D}=+8.3(\mathrm{C}=3.1$, $\left.\mathrm{CHCl}_{3}, \mathrm{~T}=24.7^{\circ} \mathrm{C}\right) .{ }^{1} \mathrm{H}-\mathrm{NMR}\left(\mathrm{CDCl}_{3}, 300 \mathrm{MHz}\right) \delta 0.024(\mathrm{~s}, 3 \mathrm{H}), 0.033(\mathrm{~s}, 3 \mathrm{H}), 0.054(\mathrm{~s}$, 3H), $0.066(\mathrm{~s}, 3 \mathrm{H}), 0.077(\mathrm{~s}, 3 \mathrm{H}), 0.089(\mathrm{~s}, 3 \mathrm{H}), 0.102(\mathrm{~s}, 3 \mathrm{H}), 0.84(\mathrm{~s}, 9 \mathrm{H}), 0.88(\mathrm{~s}, 18 \mathrm{H})$, 1.58-1.68 $(\mathrm{m}, 1 \mathrm{H}), 1.85\left(\mathrm{ddd}, 1 \mathrm{H}, J_{1}=4.98, J_{2}=9.34, J_{3}=13.39\right), 2.37\left(\mathrm{dd}, 1 \mathrm{H}, J_{1}=\right.$ 7.78, $\left.J_{2}=14\right), 2.49\left(\mathrm{dd}, 1 \mathrm{H}, J_{1}=4.36, J_{2}=14\right), 3.45-3.48(\mathrm{~m}, 1 \mathrm{H}), 3.59-3.62(\mathrm{~m}, 1 \mathrm{H})$, $3.65(\mathrm{~s}, 3 \mathrm{H}), 4.02-4.09(\mathrm{~m}, 1 \mathrm{H}), 4.10-4.16(\mathrm{~m}, 1 \mathrm{H}), 5.02(\mathrm{br} . \mathrm{d}, 1 \mathrm{H}, \mathrm{J}=8.09), 5.09(\mathrm{~s}$, 2H), 7.31-7.39 (m, 5H). ${ }^{13} \mathrm{C}-\mathrm{NMR}\left(\mathrm{CDCl}_{3}, 75 \mathrm{MHz}\right)-5.3,-5.2,-4.8,-4.7,-4.4,-4.1,18.0$, $18.1,25.8,25.9,26.0,128.1,128.2,128.4,155.9,171.2$. IR 2254m, 1719s. MS 706 (100, $\left.[\mathrm{M}+\mathrm{Na}]^{+}\right)$. HRMS calcd. for $\mathrm{C}_{34} \mathrm{H}_{65} \mathrm{NO}_{7} \mathrm{Si}{ }_{3} \mathrm{Na}(\mathrm{M}+\mathrm{Na})^{+}:$706.3961, found: 706.3954 . 
(6S)-6-Benzyloxycarbonylamino-7-(tert-butyl-dimethyl-silanyloxy)-(3R,5S)-4,5-

hydroxy-3-oxo-heptanoic acid methyl ester
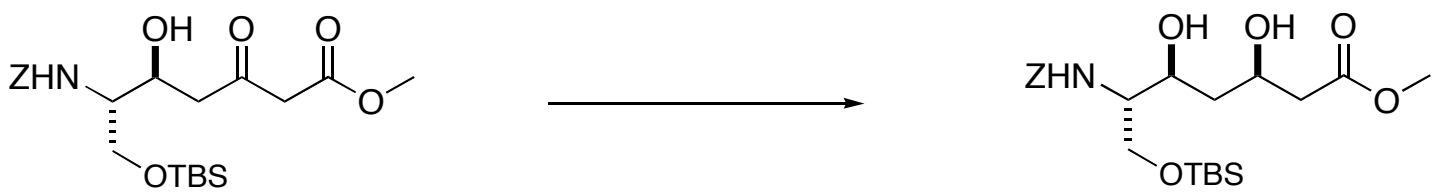

A solution of $\mathrm{Et}_{3} \mathrm{~B}(750 \mu \mathrm{l}, 0.75 \mathrm{mmol}, 1.5 \mathrm{eq}, 1 \mathrm{M}$ sol. in hexane) and pivalic acid (2.5 $\mathrm{mg}, 25 \mu \mathrm{mol}, 0.05 \mathrm{eq})$ dissolved in dry THF $(0.5 \mathrm{~mL})$ and $\mathrm{MeOH}(1 \mathrm{~mL})$ was stirred at RT for 1 hour and was then cooled to $-70^{\circ} \mathrm{C}$. (6S)-6-Benzyloxycarbonylamino-7-(tertbutyl-dimethyl-silanyloxy)-(5S)-5-hydroxy-3-oxo-heptanoic acid methyl ester (0.225 g, $0.50 \mathrm{mmol})$ in dry THF (1 mL) was added dropwise followed by $\mathrm{NaBH}_{4}(57 \mathrm{mg}, 1.5$ mmol, 3 eq) and it was stirred at $-70{ }^{\circ} \mathrm{C}$ for 4 hours. $\mathrm{H}_{2} \mathrm{O}_{2}(3 \mathrm{~mL})$ and $\mathrm{H}_{2} \mathrm{O}(5 \mathrm{~mL})$ were then added at $-70{ }^{\circ} \mathrm{C}$ and the reaction allowed to warm until RT. Then $\mathrm{CHCl}_{3}$ and $\mathrm{H}_{2} \mathrm{O}$ were added and the aqueous phase extracted with chloroform. The combined organic layers were washed with $5 \mathrm{x} \mathrm{H}_{2} \mathrm{O}$, dried over $\mathrm{Na}_{2} \mathrm{SO}_{4}$, filtered and evaporated under reduced pressure. FC (Hexane/AcOET 5.5:4.5) gave the title compound (155.8 mg, $0.34 \mu \mathrm{mol}, 69 \%) . \mathrm{R}_{\mathrm{f}}=0.31\left(\right.$ AcOEt/Hexane 3.5:6.5). $[\alpha]_{\mathrm{D}}=+10.6\left(\mathrm{C}=0.95, \mathrm{CHCl}_{3}, \mathrm{~T}=\right.$ $\left.30.7^{\circ} \mathrm{C}\right) .{ }^{1} \mathrm{H}-\mathrm{NMR}\left(\mathrm{CDCl}_{3}, 300 \mathrm{MHz}\right) \delta 0.055(\mathrm{~s}, 3 \mathrm{H}), 0.063(\mathrm{~s}, 3 \mathrm{H}), 0.88(\mathrm{~s}, 9 \mathrm{H}), 1.5-1.6$ $(\mathrm{m}, 1 \mathrm{H}), 1.67-1.82(\mathrm{~m}, 1 \mathrm{H}), 2.44-2.51(\mathrm{~m}, 2 \mathrm{H}), 3.57-3.65(\mathrm{~m}, 1 \mathrm{H}), 3.7(\mathrm{~s}, 3 \mathrm{H}), 3.72-3.77$ (br. s, 1H), 3.78-3.82 (m, 1H), 3.87-3.92 (br. s, 1H), $5.1(\mathrm{~s}, 2 \mathrm{H}), 5.38(\mathrm{~d}, 1 \mathrm{H}, J=9.03)$, 7.31-7.37 (m, 5H). ${ }^{13} \mathrm{C}-\mathrm{NMR}\left(\mathrm{CDCl}_{3}, 75 \mathrm{MHz}\right)$-5.4, 18.3, 25.9, 39.4, 41.5, 51.9, 55.0, $65.2,66.9,68.2,72.0,128.0,128.1,128.4,136.3,156.3,172.6$. IR $3611-3144 m, 1721 s$, 1510m. MS 478.2 (100, $\left.[\mathrm{M}+\mathrm{Na}]^{+}\right)$. HRMS calcd. for $\mathrm{C}_{22} \mathrm{H}_{37} \mathrm{NO}_{7} \mathrm{SiNa}(\mathrm{M}+\mathrm{Na})^{+}:$478.2232, found: 478.2224 . 
(6S)-6-Benzyloxycarbonylamino-(3R,5S)-3,5,7-tris-(tert-butyl-dimethyl-silanyloxy)heptanoic acid methyl ester<smiles>[Z]N[C@@H](C[OH2+])[C@H](O)C[C@@H](O)CC(=O)OC</smiles>

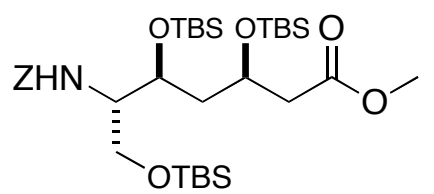

A solution of (6S)-6-Benzyloxycarbonylamino-7-(tert-butyl-dimethyl-silanyloxy)(3R,5S)-4,5-hydroxy-3-oxo-heptanoic acid methyl ester (19.0 mg, $41.7 \mu \mathrm{mol})$ in dry $\mathrm{CH}_{2} \mathrm{Cl}_{2}$ was cooled to $-20^{\circ} \mathrm{C}$ and 2,6 lutidine (19.4 $\left.\mu \mathrm{l}, 0.17 \mathrm{mmol}, 4 \mathrm{eq}\right)$ was added dropwise followed by TBSOTf $(29 \mu \mathrm{l}, 0.125 \mathrm{mmol}, 3 \mathrm{eq})$. The reaction mixture was stirred at $-20{ }^{\circ} \mathrm{C}$ for $1 \mathrm{~h} 15$. Then $\mathrm{CH}_{2} \mathrm{Cl}_{2}$ was added and the organic phase was washed $3 x$ with citric acid (10\%) and $2 x$ with $\mathrm{H}_{2} \mathrm{O}$, dried over $\mathrm{Na}_{2} \mathrm{SO}_{4}$, filtered and evaporated under reduced pressure. FC (AcOEt/hexane 1:9) gave 9 (21.0 mg, $30.7 \mu \mathrm{mol}, 75 \%$ ). $\mathrm{R}_{\mathrm{f}}$ $=0.68\left(\right.$ AcOEt/hexane 2:8). $[\alpha]_{\mathrm{D}}=-1.3\left(\mathrm{C}=1.05, \mathrm{CHCl}_{3}, \mathrm{~T}=25.5^{\circ} \mathrm{C}\right) .{ }^{1} \mathrm{H}-\mathrm{NMR}\left(\mathrm{CDCl}_{3}\right.$, $300 \mathrm{MHz}) \delta 0.056(\mathrm{~s}, 6 \mathrm{H}), 0.062$ (s, 3H), $0.07,0.083(\mathrm{~s}, 6 \mathrm{H}), 0.098(\mathrm{~s}, 3 \mathrm{H}), 0.86(\mathrm{~s}, 9 \mathrm{H})$, $0.88(\mathrm{~s}, 9 \mathrm{H}), 0.89(\mathrm{~s}, 9 \mathrm{H}), 1.63-1.84(\mathrm{~m}, 2 \mathrm{H}), 2.43(\mathrm{~d}, 2 \mathrm{H}, \mathrm{J}=6.22), 3.52\left(\mathrm{dd}, 1 \mathrm{H}, J_{1}=\right.$ 7.79, $\left.J_{2}=9.03\right), 3.62(s, 3 H), 3.66(d, 1 H, J=5), 3.69(s, 1 H), 3.73(m, 1 H), 4.99$ (br.d, $1 \mathrm{H}, J=8.7), 5.1(\mathrm{~s}, 2 \mathrm{H}), 7.3-7.39(\mathrm{~m}, 5 \mathrm{H}) .{ }^{13} \mathrm{C}-\mathrm{NMR}\left(\mathrm{CDCl}_{3}, 75 \mathrm{MHz}\right)$-5.1, -4.5, -4.0, 18.0, 18.2, 18.6, 26.0, 26.1, 26.2, 42.1, 43.1, 51.6, 55.1, 62.0, 66.7, 66.9, 67.4, 128.3, 128.4, 128.7, 156.4, 171.6. IR 1727m. MS 706.3 (100). HRMS calcd. for $\mathrm{C}_{34} \mathrm{H}_{65} \mathrm{NO}_{7} \mathrm{Si}_{3} \mathrm{Na}(\mathrm{M}+\mathrm{Na})^{+}:$706.3961, found: 706.3960 . 


\section{7-Benzyloxy-(6S)-6-benzyloxycarbonylamino-(3S, 5R)-3,5-bis-(tert-butyl-dimethyl-}

silanyloxy)-heptanoic acid methyl ester<smiles>CNC(CO)C(O)CC(=O)CC(=O)OC</smiles><smiles>CNC(CO)C(O)CC(O)CC(=O)OC</smiles>

A solution of $\mathrm{Et}_{3} \mathrm{~B}(1.08 \mathrm{~mL}, 1.08 \mathrm{mmol}, 1.5 \mathrm{eq}, 1 \mathrm{M}$ sol. in hexane) and pivalic acid (3.7 $\mathrm{mg}, 36 \mu \mathrm{mol}, 0.05 \mathrm{eq})$ dissolved in dry THF $(0.5 \mathrm{~mL})$ and $\mathrm{MeOH}(1.5 \mathrm{~mL})$ was stirred at RT for 1hour and was then cooled to $-70{ }^{\circ} \mathrm{C}$. 7-Benzyloxy-(6S)-6benzyloxycarbonylamino-(5R)-5-hydroxy-3-oxo-heptanoic acid methyl ester (300 mg, $0.72 \mathrm{mmol})$ in dry THF (2.2 mL) was added dropwise followed by $\mathrm{NaBH}_{4}(82 \mathrm{mg}, 2.16$ mmol, 3 eq) and it was stirred at $-70{ }^{\circ} \mathrm{C}$ for 4hours. $\mathrm{H}_{2} \mathrm{O}_{2}(3 \mathrm{~mL})$ and $\mathrm{H}_{2} \mathrm{O}(5 \mathrm{~mL})$ were then added at $-70{ }^{\circ} \mathrm{C}$ and the reaction was allowed to warm to RT. Then $\mathrm{CHCl}_{3}$ and $\mathrm{H}_{2} \mathrm{O}$ were added and the aqueous phase extracted with chloroform. The combined organic layers were washed with $5 \mathrm{x} \mathrm{H}_{2} \mathrm{O}$, dried over $\mathrm{Na}_{2} \mathrm{SO}_{4}$, filtered and evaporated under reduced pressure. It was directly introduced in the next step.

It was dissolved in dry $\mathrm{CH}_{2} \mathrm{Cl}_{2}(1.5 \mathrm{~mL})$ and cooled to $-20{ }^{\circ} \mathrm{C}$. Then 2,6-lutidine $(0.335$ $\mathrm{mL}, 2.88 \mathrm{mmol}, 4 \mathrm{eq})$ and TBSOTf ( $496 \mu \mathrm{l}, 2.16 \mathrm{mmol}, 3 \mathrm{eq})$ were added dropwise and the reaction mixture stirred at this temperature for 3 hours. It was washed $3 x$ with citric acid (10\%), 2x with $\mathrm{H}_{2} \mathrm{O}$, filtered and evaporated under reduced pressure. FC (hexane/AcOEt 9:1) gave the title compound 7 (395 mg, $0.6 \mathrm{mmol}, 85 \%) . \mathrm{R}_{\mathrm{f}}=0.64$ (AcOEt/hexane 2:8). $[\alpha]_{\mathrm{D}}=-24.9\left(\mathrm{C}=0.9, \mathrm{CHCl}_{3}, \mathrm{~T}=30.1{ }^{\circ} \mathrm{C}\right) .{ }^{1} \mathrm{H}-\mathrm{NMR}\left(\mathrm{CDCl}_{3}, 300\right.$ $\mathrm{MHz}) \delta 0.015(\mathrm{~s}, 3 \mathrm{H}), 0.03(\mathrm{~s}, 3 \mathrm{H}), 0.07(\mathrm{~s}, 3 \mathrm{H}), 0.099(\mathrm{~s}, 3 \mathrm{H}), 0.84(\mathrm{~s}, 9 \mathrm{H}), 0.86(\mathrm{~s}, 9 \mathrm{H})$, 1.67-1.8 (m, 2H), 2.35-2.45 (m, 1H), 2.48-2.55 (m, 1H), $3.57(\mathrm{~d}, 1 \mathrm{H}, J=5.2), 3.63(\mathrm{~s}$, 3H), 3.9-4.01 (m, 2H), 4.2-4.28 (m, 1H), $4.5(\mathrm{~s}, 2 \mathrm{H}), 4.92-5(\mathrm{~m}, 1 \mathrm{H}), 5.1(\mathrm{~s}, 2 \mathrm{H}), 7.2-7.4$ 
$(m, 10 \mathrm{H})$. IR 1735m, 1501w. MS $682.4\left(100,[\mathrm{M}+\mathrm{Na}]^{+}\right)$. HRMS calcd. for $\mathrm{C}_{35} \mathrm{H}_{57} \mathrm{NO}_{7} \mathrm{Si} 2 \mathrm{Na}(\mathrm{M}+\mathrm{Na})^{+}: 682.3566$, found: 682.3558 .

(6S)-6-(2-benzyloxy-benzoylamino)-(3R,5S)-3,5,7-tris-(tert-butyl-dimethylsilanyloxy)-heptanoic acid methyl ester
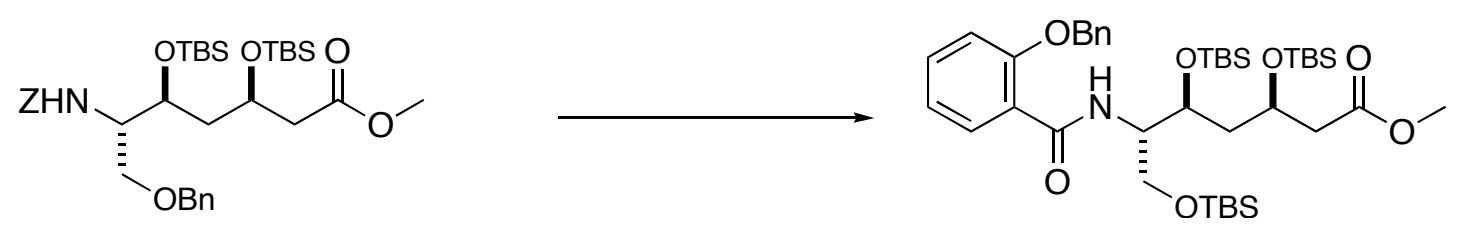

To a solution of (6S)-6-Benzyloxycarbonylamino-(3R,5S)-3,5,7-tris-(tert-butyl-dimethylsilanyloxy)-heptanoic acid methyl ester $(70 \mathrm{mg}, 102 \mu \mathrm{mol})$ in $\mathrm{MeOH}(1 \mathrm{~mL})$ previously 3x flushed with argon was added $\mathrm{Pd} / \mathrm{C}$. Then it was flushed with $\mathrm{H}_{2}$ and it was stirred at $\mathrm{RT}$ for 4 hours. $\mathrm{Pd} / \mathrm{C}$ was filtered over celite and the solvent was removed under reduced pressure. This product was added to a solution of $\mathrm{O}-\mathrm{Bn}$-salicylic acid (27.9 $\mathrm{mg}, 122 \mu \mathrm{mol}, 1.2 \mathrm{eq})$ dissolved in dry $\mathrm{CHCl}_{3}(1.5 \mathrm{~mL})$ with EDC. $\mathrm{HCl}(23.4 \mathrm{mg}, 122$ $\mu \mathrm{mol}, 1.2 \mathrm{eq}), \mathrm{HOBt}(18.9 \mathrm{mg}, 122 \mu \mathrm{mol}, 1.2 \mathrm{eq})$ and NMM (34 $\mu \mathrm{L}, 306 \mu \mathrm{mol}, 3 \mathrm{eq})$. The reaction mixture was stirred at $\mathrm{RT}$ for 20 hours. The solvent was then removed under reduced pressure and the residue taken up with AcOEt. It was washed 3x with citric acid $(10 \%)$ and $3 x$ with satd. $\mathrm{NaHCO}_{3}$. The organic phase was dried over $\mathrm{MgSO}_{4}$, filtered and evaporated under reduced pressure. FC (AcOEt/Hexane 1:9) gave 13 (45 mg, $59 \mu \mathrm{mol}, 58 \%) . \mathrm{R}_{\mathrm{f}}=0.43\left(\mathrm{AcOEt} /\right.$ Hexane 2:8). $[\alpha]_{\mathrm{D}}=-4.2\left(\mathrm{C}=2.235, \mathrm{CHCl}_{3}, \mathrm{~T}=\right.$ $\left.26.5^{\circ} \mathrm{C}\right) .{ }^{1} \mathrm{H}-\mathrm{NMR}\left(\mathrm{CDCl}_{3}, 300 \mathrm{MHz}\right) \delta 0.061(\mathrm{~s}, 6 \mathrm{H}), 0.071(\mathrm{~s}, 3 \mathrm{H}), 0.091(\mathrm{~s}, 3 \mathrm{H}), 0.094$ (s, 3H), 0.105 (s, 3H), $0.84(\mathrm{~s}, 9 \mathrm{H}), 0.86(\mathrm{~s}, 9 \mathrm{H}), 0.89(\mathrm{~s}, 9 \mathrm{H}), 1.67-1.78(\mathrm{~m}, 2 \mathrm{H}), 2.35-$ $2.48(\mathrm{~m}, 2 \mathrm{H}), 3.49(\mathrm{~s}, 3 \mathrm{H}), 3.6\left(\mathrm{dd}, 1 \mathrm{H}, J_{1}=8.4, J_{2}=9.33\right), 3.71\left(\mathrm{dd}, 1 \mathrm{H}, J_{1}=5.6, J_{2}=\right.$ 9.6), 4.1-4.21 (m, 2H), 4.23-4.28 (m, 1H), $5.29(\mathrm{~s}, 2 \mathrm{H}), 6.91\left(\mathrm{dd}, 1 \mathrm{H}, J_{1}=0.6, J_{2}=8.4\right)$, 7.0-7.1 (m, 1H), 7.28-7.37 (m, 6H), $8.0(\mathrm{~d}, 1 \mathrm{H}, J=8.4), 8.16\left(\mathrm{dd}, 1 \mathrm{H}, J_{1}=1.87, J_{2}=7.8\right)$. 
${ }^{13} \mathrm{C}-\mathrm{NMR}\left(\mathrm{CDCl}_{3}, 75 \mathrm{MHz}\right)-5.1,-4.5,-4.2,-4.0,17.9,18.1,18.5,25.9,26.0,26.1,42.1$, 43.2, 51.4, 53.8, 61.2, 66.5, 67.1, 70.7, 113.2, 121.3, 122.3, 126.7, 128.1, 128.7, 132.2, 132.3, 135.9, 156.3, 164.9, 171.3. IR 2953m, 2928m, 2856m, 1740m, 1654m. MS 760 (100, $\left.[\mathrm{M}+\mathrm{H}]^{+}\right)$. HRMS calcd. for $\mathrm{C}_{40} \mathrm{H}_{69} \mathrm{NO}_{7} \mathrm{Si} \mathrm{i}_{3} \mathrm{Na}(\mathrm{M}+\mathrm{Na})^{+}:$782.4274, found: 782.4285 .

\section{(6S)-6-(2-benzyloxy-benzoylamino)-(3S,5S)-3,5,7-tris-(tert-butyl-dimethyl-} silanyloxy)-heptanoic acid methyl ester
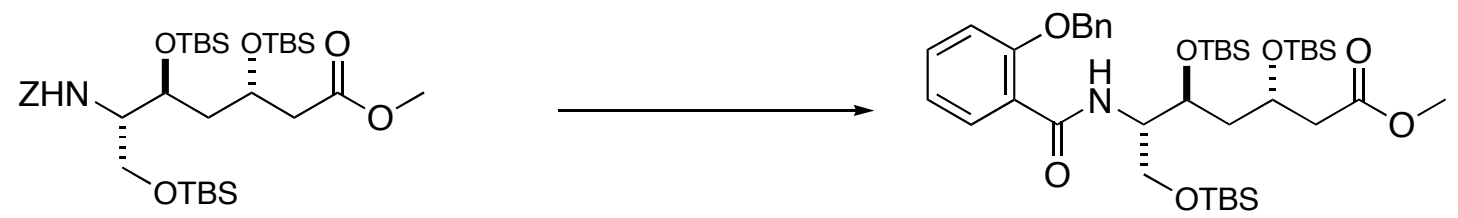

To a solution of (6S)-6-Benzyloxycarbonylamino-(3S,5S)-3,5,7-tris-(tert-butyl-dimethylsilanyloxy)-heptanoic acid methyl ester $(59 \mathrm{mg}, 86 \mu \mathrm{mol})$ in $\mathrm{MeOH}(0.7 \mathrm{~mL})$ previously 3x flushed with argon was added $\mathrm{Pd} / \mathrm{C}(1 \mathrm{mg}, 10 \%)$. Then it was flushed with $\mathrm{H}_{2}$ and it was stirred at RT for 4 hours. $\mathrm{Pd} / \mathrm{C}$ was filtered over celite and the solvent was removed under reduced pressure. This product was added to a solution of $\mathrm{O}-\mathrm{Bn}$ salicylic acid (19.6 mg, $86 \mu \mathrm{mol}, 1 \mathrm{eq})$ dissolved in dry $\mathrm{CH}_{2} \mathrm{Cl}_{2}(1 \mathrm{~mL})$ with $\mathrm{EDC} . \mathrm{HCl}$ (16.5 mg, $86 \mu \mathrm{mol}, 1 \mathrm{eq}), \mathrm{HOBt}(13 \mathrm{mg}, 86 \mu \mathrm{mol}, 1 \mathrm{eq})$ and NMM (28 $\mu \mathrm{L}, 258 \mu \mathrm{mol}, 3$ eq). The reaction mixture was stirred at RT for 20 hours. The solvent was then removed under reduced pressure and the residue taken up with AcOEt. It was washed 3x with citric acid (10 \%) and $3 \mathrm{x}$ with satd. $\mathrm{NaHCO}_{3}$. The organic phase was dried over $\mathrm{MgSO}_{4}$, filtered and evaporated under reduced pressure. FC (AcOEt/hexane 1:9) gave 12 (24 $\mathrm{mg}, 32 \mu \mathrm{mol}, 70 \%) . \mathrm{R}_{\mathrm{f}}=0.55\left(\right.$ AcOEt/hexane 2:8). $[\alpha]_{\mathrm{D}}=+26.3\left(\mathrm{C}=0.9, \mathrm{CHCl}_{3}, \mathrm{~T}=\right.$ $\left.22.2^{\circ} \mathrm{C}\right) .{ }^{1} \mathrm{H}-\mathrm{NMR}\left(\mathrm{CDCl}_{3}, 300 \mathrm{MHz}\right) \delta-0.06(\mathrm{~s}, 3 \mathrm{H}),-0.01(\mathrm{~s}, 3 \mathrm{H}), 0.06(\mathrm{~s}, 6 \mathrm{H}), 0.07(\mathrm{~s}$, 3H), $0.1(\mathrm{~s}, 3 \mathrm{H}), 0.8(\mathrm{~s}, 9 \mathrm{H}), 0.81(\mathrm{~s}, 9 \mathrm{H}), 0.9(\mathrm{~s}, 9 \mathrm{H}), 1.65\left(\mathrm{ddd}, 1 \mathrm{H}, J_{1}=4.67, J_{2}=9.03\right.$, $\left.J_{3}=13.69\right), 1.89$ (ddd, $\left.1 \mathrm{H}, J_{1}=4.67, J_{2}=9.34, J_{3}=13.7\right), 2.36\left(\mathrm{dd}, 1 \mathrm{H}, J_{1}=8.09, J_{2}=\right.$ 
14.01), $2.52\left(\mathrm{dd}, 1 \mathrm{H}, J_{1}=4.05, J_{2}=14.01\right), 3.50(\mathrm{t}, 1 \mathrm{H}, J=9.34), 3.64(\mathrm{~s}, 3 \mathrm{H}), 3.67$ (dd, $\left.J_{1}=4.98, J_{2}=9.34\right), 4.11-4.24(\mathrm{~m}, 3 \mathrm{H}), 5.3(\mathrm{~s}, 2 \mathrm{H}), 6.90\left(\mathrm{dd}, 1 \mathrm{H}, J_{1}=0.62, J_{2}=8.4\right)$, $7.03\left(\mathrm{td}, 1 \mathrm{H}, J_{1}=0.93, J_{2}=7.78\right), 7.29-7.36(\mathrm{~m}, 5 \mathrm{H}), 8.0$ (br. $\left.\mathrm{d}, \mathrm{J}=8.4\right), 8.16\left(\mathrm{dd}, 1 \mathrm{H}, J_{1}\right.$ $\left.=1.87, J_{2}=7.78\right) .{ }^{13} \mathrm{C}-\mathrm{NMR}\left(\mathrm{CDCl}_{3}, 75 \mathrm{MHz}\right)-5.2,-5.0,-4.8,-4.6,-4.3,-4.1,18.0,18.2$, $18.3,25.9,26.1,42.4,42.5,51.6,53.6,60.7,66.8,67.0,71.0,113.5,121.6,122.7$, 126.9, 128.4, 129.0, 132.7, 136.4, 156.7, 165.4, 171.7. IR 3399s, 1735m, 1647m. MS $782\left(100,[\mathrm{M}+\mathrm{Na}]^{+}\right)$. HRMS calcd. for $\mathrm{C}_{40} \mathrm{H}_{69} \mathrm{NO}_{7} \mathrm{Si}_{3} \mathrm{Na}(\mathrm{M}+\mathrm{Na})^{+}:$782.4274, found: 782.4285.

(6S)-6-(2-benzyloxy-benzoylamino)-(3S,5R)-3,5,7-tris-(tert-butyl-dimethylsilanyloxy)-heptanoic acid methyl ester
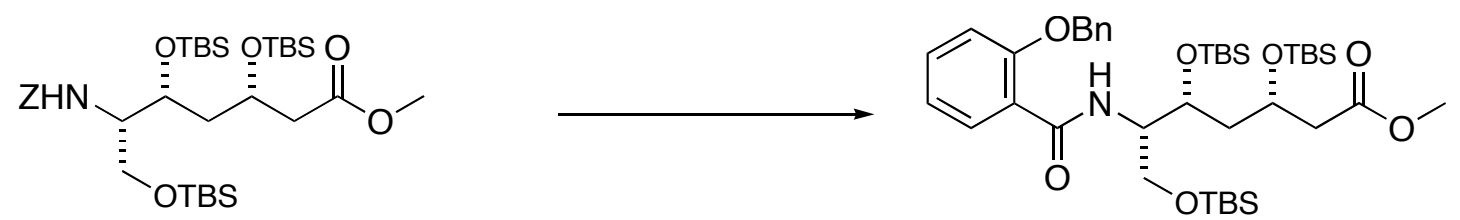

To a solution of 7-Benzyloxy-(6S)-6-benzyloxycarbonylamino-(3S, 5R)-3,5-bis-(tertbutyl-dimethyl-silanyloxy)-heptanoic acid methyl ester (58 mg, $88 \mu \mathrm{mol})$ in $\mathrm{MeOH}(1$ $\mathrm{mL}$ ) previously $3 x$ flushed with argon was added $\mathrm{Pd} / \mathrm{C}$. Then it was flushed with $\mathrm{H}_{2}$ and it was stirred at RT for 4 hours. Pd/C was filtered over celite and the solvent was removed under reduced pressure. This product was added to a solution of $\mathrm{O}-\mathrm{Bn}$ salicylic acid $(20 \mathrm{mg}, 88 \mu \mathrm{mol}, 1 \mathrm{eq})$ dissolved in dry $\mathrm{CH}_{2} \mathrm{Cl}_{2}(1 \mathrm{~mL})$ with EDC. $\mathrm{HCl}(17$ mg, $88 \mu \mathrm{mol}, 1$ eq), HOBt (13.5 mg, $88 \mu \mathrm{mol}, 1$ eq) and NMM (29 $\mu \mathrm{L}, 264 \mu \mathrm{mol}, 3 \mathrm{eq})$. The reaction mixture was stirred at RT for 20 hours. The solvent was then removed under reduced pressure and the residue taken up with AcOEt. It was washed $3 x$ with citric acid (10\%) and $3 \mathrm{x}$ with satd. $\mathrm{NaHCO}_{3}$. The organic phase was dried over $\mathrm{MgSO}_{4}$, filtered and the solvent evaporated under reduced pressure. It was dissolved in $\mathrm{CH}_{2} \mathrm{Cl}_{2}$ 
$(1 \mathrm{~mL})$ and cooled to $-20^{\circ} \mathrm{C}$. Then TBSOTf $(44.5 \mu \mathrm{l}, 194 \mu \mathrm{mol}, 2.2 \mathrm{eq})$ and 2,6-lutidine (22.5 $\mu \mathrm{l}, 194 \mu \mathrm{mol}, 2.2 \mathrm{eq})$ were added dropwise. The reaction mixture was stirred at $-20{ }^{\circ} \mathrm{C}$ for 3 hours. It was washed $2 \mathrm{x}$ with citric acid, dried over $\mathrm{Na}_{2} \mathrm{SO}_{4}$, filtered and evaporated under reduced pressure. FC (AcOEt/hexane 1:7) gave 11 (44 mg, 58 umol, $66 \%) \cdot R_{f}=0.33\left(\right.$ AcOEt/hexane 1:7). $[\alpha]_{D}=-0.90\left(\mathrm{C}=1.56, \mathrm{CHCl}_{3}, \mathrm{~T}=25^{\circ} \mathrm{C}\right) .{ }^{1} \mathrm{H}-\mathrm{NMR}$ $\left(\mathrm{CDCl}_{3}, 300 \mathrm{MHz}\right) \delta-0.042$ (s, 3H), -0.037 (s, 3H), 0.001 (s, 6H), 0.024 (s, 3H), 0.05 (s, $3 \mathrm{H}), 0.80(\mathrm{~s}, 9 \mathrm{H}), 0.83(\mathrm{~s}, 9 \mathrm{H}), 0.84(\mathrm{~s}, 9 \mathrm{H}), 1.55-1.61(\mathrm{~m}, 2 \mathrm{H}), 2.29\left(\mathrm{dd}, 1 \mathrm{H}, J_{1}=9.03, J_{2}\right.$ $=14.63), 2.57\left(\mathrm{dd}, J_{1}=3.11, J_{2}=14.63\right), 3.5-3.6(\mathrm{~m}, 1 \mathrm{H}), 3.64(\mathrm{~s}, 3 \mathrm{H}), 3.64-3.73(\mathrm{~m}$, $1 \mathrm{H}), 3.84-3.91(\mathrm{~m}, 1 \mathrm{H}), 5.18(\mathrm{~s}, 2 \mathrm{H}), 7.0(\mathrm{~d}, 1 \mathrm{H}, J=7.8), 7.08\left(\mathrm{td}, J_{1}=0.93, J_{2}=7.8\right)$, 7.35-7.47 (m, 5H), $7.9(\mathrm{~d}, 1 \mathrm{H}, J=8.09), 8.23\left(\mathrm{dd}, 1 \mathrm{H}, J_{1}=1.87, J_{2}=7.8\right) .{ }^{13} \mathrm{C}-\mathrm{NMR}$ $\left(\mathrm{CDCl}_{3}, 75 \mathrm{MHz}\right)-5.26,-5.14,-4.95,-4.35,-4.15,18.0,25.8,26.0,41.2,42.9,51.4$, $55.3,61.2,67.0,68.4,71.2,112.5,121.451,121.945,127.7,128.5,128.8,132.5,135.5$, 156.5, 164.6, 171.9. IR 3392w, 1739m, 1656m. MS $760\left(100,[\mathrm{M}+\mathrm{H}]^{+}\right)$.

\section{(6S)-6-(2-benzyloxy-benzoylamino)-(3R,5S)-3,5,7-tris-(tert-butyl-dimethyl-}

\section{silanyloxy)-heptanoic acid}<smiles>COC(=O)CC(O)CC(O)C(CO)NC(=O)c1ccccc1O</smiles>

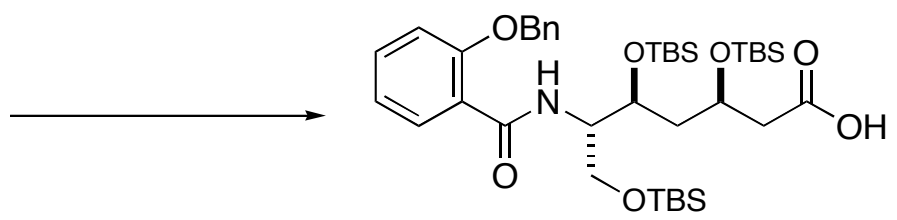

To a solution of (6S)-6-(2-benzyloxy-benzoylamino)-(3R,5S)-3,5,7-tris-(tert-butyldimethyl-silanyloxy)-heptanoic acid methyl ester (44 mg, $57.9 \mu \mathrm{mol})$ in $\mathrm{MeOH}(0.6 \mathrm{~mL})$ cooled at $0{ }^{\circ} \mathrm{C}$ was added $\mathrm{NaOH}(58 \mu \mathrm{l}, 174 \mu \mathrm{mol}, 1 \mathrm{M}, 3 \mathrm{eq})$. The reaction mixture was stirred 1 hour at RT and then one night at RT. As a TLC at this point showed no total conversion, THF $(0.3 \mathrm{~mL})$ and $\mathrm{NaOH}(1 \mathrm{M}, 39 \mu \mathrm{l}, 116 \mu \mathrm{mol}, 2$ eq) were added and after 6 hours the reaction was completed. The solvent was evaporated down under reduced 
pressure and the residue acidified to $\mathrm{pH}=1$ with $\mathrm{HCl}(1 \mathrm{M})$. It was then extracted 3 times with AcOEt and the combined organic phases were dried over $\mathrm{MgSO}_{4}$, filtered, and evaporated under reduced pressure. It was directly used without further purification in the next step.

(3R, 5S, 6S)-6-(Benzyloxy-benzoylamino)-3,5,7-tris-(tert-butyl-dimethyl-silanoxy)heptanoic acid-L-Thr(OBn)-D-Ser(OBn)-L-Ser(OBn)-(3S)-THQ(OBn)

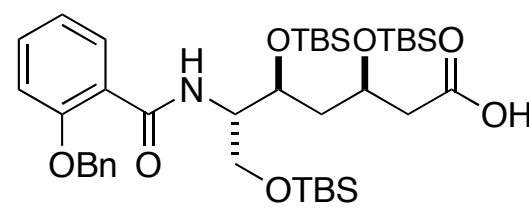

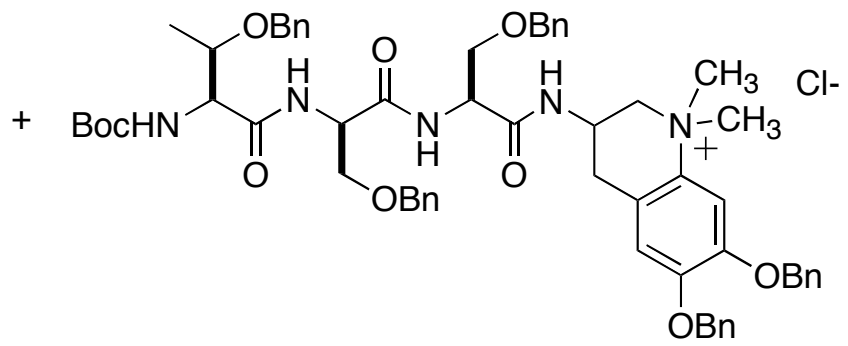

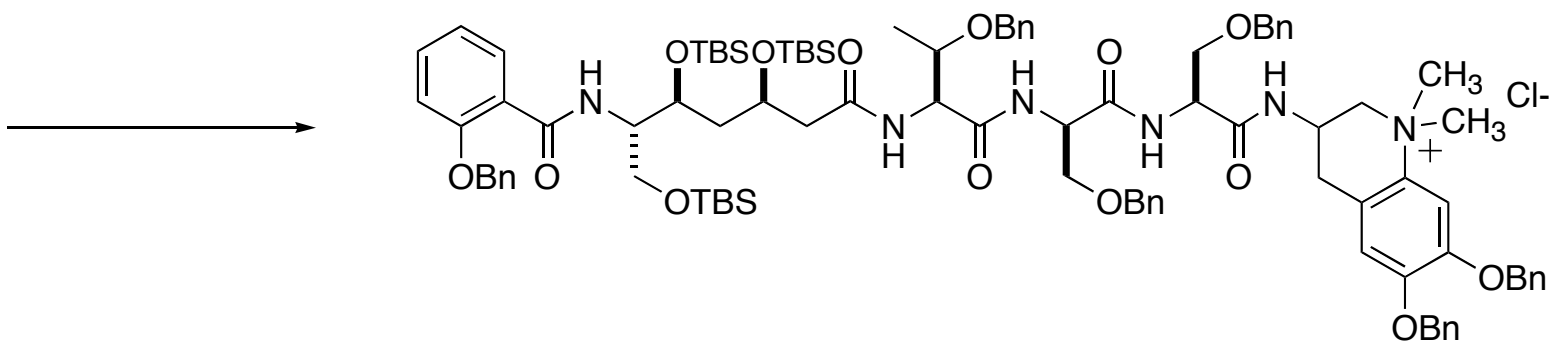

Boc-L-Thr(OBn)-L-Ser(OBn)-L-Ser(OBn)-(3S)-THQ(OBn) (36 mg, $33.6 \mu \mathrm{mol}$ ) was dissolved in dioxane $(0.6 \mathrm{~mL})$ and $\mathrm{HCl}$.dioxane $(4 \mathrm{M}, 100 \mu \mathrm{l}, 400 \mu \mathrm{mol})$ was added at 0 ${ }^{\circ} \mathrm{C}$. It was stirred 1 hour at this temperature and 3 hours at RT. The solvent was then removed under reduced pressure and the product dried under high vacuum 2 hours. It was used without further purification.

To a solution of (6S)-6-(2-benzyloxy-benzoylamino)-(3R,5S)-3,5,7-tris-(tert-butyldimethyl-silanyloxy)-heptanoic acid ( $30 \mathrm{mg}, 40.3 \mu \mathrm{mol}, 1.2 \mathrm{eq})$ in DMF (0.5 mL) at -18 ${ }^{\circ} \mathrm{C}$ was added iBuOCOCl (5.3 $\left.\mu \mathrm{l}, 40.3 \mu \mathrm{mol}, 1.2 \mathrm{eq}\right)$ and NMM (4.4 $\left.\mu \mathrm{l}, 40.3 \mu \mathrm{mol}, 2 \mathrm{eq}\right)$. The reaction mixture was stirred at this temperature for $20 \mathrm{~min}$. Then L-Thr(OBn)-L- 
Ser(OBn)-L-Ser(OBn)-(3S)-THQ(OBn) (33.8 mg, $33.6 \mu \mathrm{mol}, 1$ eq) dissolved in DMF (0.5 $\mathrm{mL})$ and NMM (4.4 $\mu \mathrm{l}, 40.3 \mu \mathrm{mol}, 2$ eq) was added dropwise at $-18{ }^{\circ} \mathrm{C}$ and it was allowed to warm over 4 hours. The solvent was evaporated under reduced pressure and the residue taken up in AcOEt. It was washed $3 x$ with $\mathrm{H}_{2} \mathrm{O}$, and the resulting water extracted $3 x$ with AcOEt. The combined organic phases were dried over $\mathrm{Na}_{2} \mathrm{SO}_{4}$, filtered and evaporated under reduced pressure. $\mathrm{FC}\left(\mathrm{CH}_{2} \mathrm{Cl}_{2} / \mathrm{MeOH} 10: 1\right)$ gave the title compound (31 mg, $18.3 \mathrm{mmol}, 54 \%)$. Colorless oil. $\mathrm{R}_{\mathrm{f}}=0.18\left(\mathrm{CH}_{2} \mathrm{Cl}_{2} / \mathrm{MeOH} 10: 1\right)$. $[\alpha]_{\mathrm{D}}$ $=+7.9\left(\mathrm{C}=0.55, \mathrm{CHCl}_{3}, \mathrm{~T}=26{ }^{\circ} \mathrm{C}\right) .{ }^{1} \mathrm{H}-\mathrm{NMR}\left(\mathrm{CDCl}_{3}, 300 \mathrm{MHz}\right) 0.013(\mathrm{~s}, 3 \mathrm{H}), 0.032(\mathrm{~s}$, 6H), 0.061 (s, 6H), 0.079 (s, 3H), $0.80(\mathrm{~s}, 9 \mathrm{H}), 0.83(\mathrm{~s}, 9 \mathrm{H}), 0.86(\mathrm{~s}, 9 \mathrm{H}), 1.15(\mathrm{~d}, 3 \mathrm{H}, J=$ 6.54), 1.74-1.9 (m, 8H), $2.24\left(\mathrm{dd}, 1 \mathrm{H}, J_{1}=6.54, J_{2}=14.6\right), 2.35\left(\mathrm{dd}, 1 \mathrm{H}, J_{1}=3.1, J_{2}=\right.$ 14.6), $2.86\left(\mathrm{dd}, 1 \mathrm{H}, J_{1}=5.0, J_{2}=16.2\right), 3.18\left(\mathrm{dd}, 1 \mathrm{H}, J_{1}=11.5, J_{2}=16.2\right), 3.41(\mathrm{~s}, 3 \mathrm{H})$, $3.44(\mathrm{~s}, 3 \mathrm{H}), 3.56-3.74(\mathrm{~m}, 6 \mathrm{H}), 3.86-3.94(\mathrm{~m}, 2 \mathrm{H}), 4.0-4.08(\mathrm{~m}, 1 \mathrm{H}), 4.1-4.18(\mathrm{~m}, 1 \mathrm{H})$, 4.18-4.28 (m, 2H), $4.41(\mathrm{~s}, 2 \mathrm{H}), 4.42(\mathrm{~s}, 2 \mathrm{H}), 4.48(\mathrm{~s}, 2 \mathrm{H}), 4.52-4.62(\mathrm{~m}, 2 \mathrm{H}), 4.64-4.74$ (m, 1H), $5.13(\mathrm{~d}, 2 \mathrm{H}, J=2.5), 5.21(\mathrm{~d}, 2 \mathrm{H}, J=1.9), 5.26(\mathrm{~s}, 2 \mathrm{H}), 6.6(\mathrm{~s}, 1 \mathrm{H}), 6.86-6.94$ (m, 3H), 7.4-7.46 (m, 36H), 7.84 (d, 1H, J = 7.5), 7.97 (d, $J=8.7), 8.07$ (dd, $J_{1}=1.9, J_{2}=$ 7.5), $8.38(\mathrm{~m}, 2 \mathrm{H})$. IR $1653 \mathrm{~m}, 1520 \mathrm{~m}, 1216 \mathrm{~s} . \mathrm{MS} 1662.8\left(100,[\mathrm{M}+\mathrm{H}]^{+}\right)$. HRMS calcd. for $\mathrm{C}_{95} \mathrm{H}_{129} \mathrm{~N}_{6} \mathrm{O}_{14} \mathrm{Si}_{3}(\mathrm{M}+\mathrm{H})^{+}:$1661.8869, found: 1661.8897 . 


\section{Anachelin H}
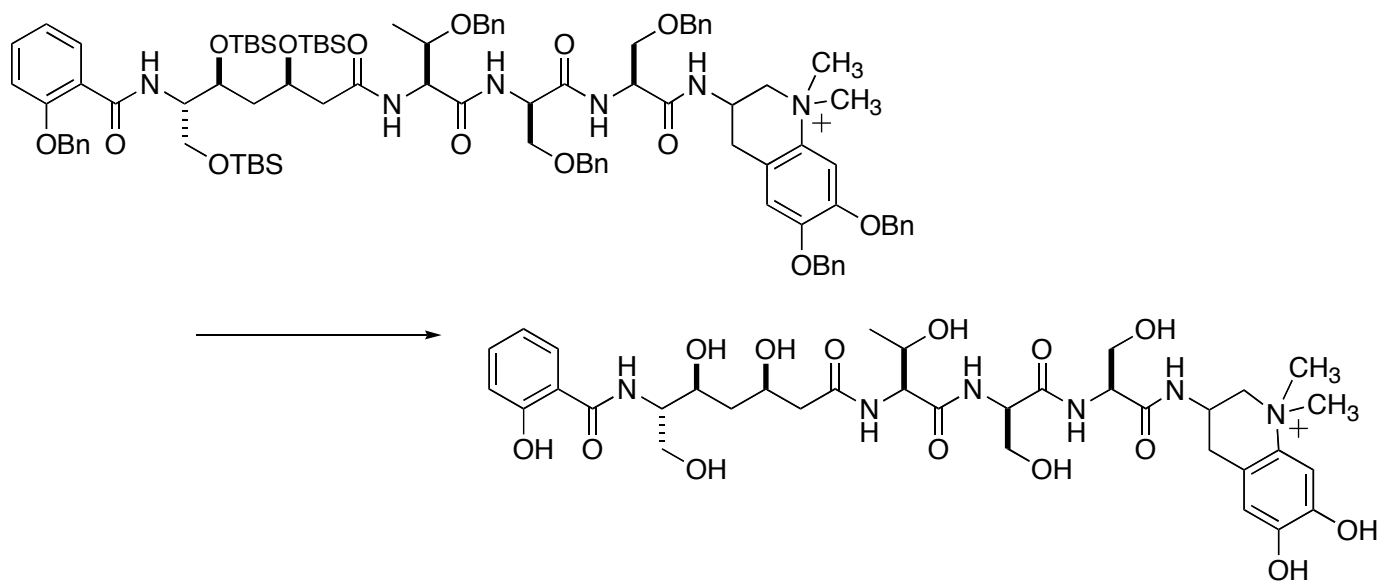

(3R, 5S, 6S)-6-(Benzyloxy-benzoylamino)-3,5,7-tris-(tert-butyl-dimethyl-silanoxy)heptanoic acid-L-Thr(OBn)-D-Ser(OBn)-L-Ser(OBn)-(3S)-THQ(OBn) (10 mg, $5.89 \mu \mathrm{mol})$ was dissolved in $\mathrm{CH}_{3} \mathrm{OH}(400 \mu \mathrm{l})$ and glacial acetic acid (30 drops) was added. The reaction vessel was flushed three times with $\mathrm{Ar}$, and then $\mathrm{Pd} / \mathrm{C}(10 \%, 8 \mathrm{mg})$ was added. The reaction vessel was three times flushed with $\mathrm{H}_{2}$ and the reaction mixture was stirred 2.5 hours at RT. The reaction mixture was then filtered over celite and the solvent removed under reduced pressure. The residue was dissolved in cold $\mathrm{MeOH}$ $(\mathrm{HCl} 1 \%)(1 \mathrm{~mL})$ and the solution stirred for 4 hours at $0{ }^{\circ} \mathrm{C}$. Then the solvent was evaporated under reduced pressure and the residual acid was coevaporated three times with chloroform. The residue was dissolved in water and lyophilized to give anachelin $\mathrm{H}$ (1) (3.3 mg, 70\%). Light yellow fluffy powder. ${ }^{1} \mathrm{H}-\mathrm{NMR}\left(\mathrm{D}_{2} \mathrm{O}, 300 \mathrm{MHz}\right) 1.18$ $(\mathrm{d}, 3 \mathrm{H}, J=6.2), 1.6-1.64(\mathrm{~m}, 1 \mathrm{H}), 1.65-1.74(\mathrm{~m}, 1 \mathrm{H}), 1.99-2.08(\mathrm{~m}, 1 \mathrm{H}), 2.27-2.35(\mathrm{~m}$, 1H), 2.89-3.04 (m, 1H), 3.05-3.15 (m, 1H), 3.36-3.44 (m, 1H), $3.53(\mathrm{~s}, 6 \mathrm{H}), 3.66-4.1(\mathrm{~m}$, $7 \mathrm{H}), 4.1-4.17(\mathrm{~m}, 2 \mathrm{H}), 4.2-4.33(\mathrm{~m}, 2 \mathrm{H}), 4.38-4.45(\mathrm{~m}, 3 \mathrm{H}), 4.53-4.7(\mathrm{~m}, 1 \mathrm{H}), 6.75(\mathrm{~s}, 1 \mathrm{H})$, 6.96-7.07 (m, 3H), 7.42-7.53 (m, 1H), $7.79(\mathrm{~d}, 1 \mathrm{H}, J=7.5)$. MS $779\left(100,[\mathrm{M}]^{+}\right)$, HRMS calcd. for $\mathrm{C}_{35} \mathrm{H}_{51} \mathrm{~N}_{6} \mathrm{O}_{14}(\mathrm{M})^{+}:$:779.3463, found: 779.3471 . 


\section{[1-(tert-Butyl-dimethyl-silanyloxymethyl)-3-(2,2-dimethyl-6-oxo-6H-[1,3]dioxin-4-}

yl)-((3R)-2-hydroxy-propyl]-(2R)carbamic acid benzyl ester

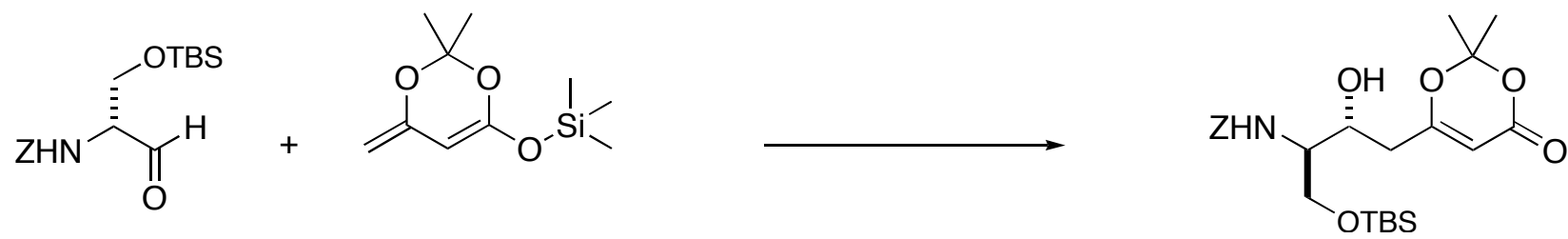

A solution of [1-(tert-Butyl-dimethyl-silanyloxymethyl)-(2R)-2-oxo-ethyl]-carbamic acid benzyl ester $(338 \mathrm{mg}, 1.08 \mathrm{mmol})$ in dry $\mathrm{CH}_{2} \mathrm{Cl}_{2}(1.5 \mathrm{~mL})$ was cooled to $0{ }^{\circ} \mathrm{C}$ and $\mathrm{Eu}(\mathrm{fod})_{3}(224 \mathrm{mg}, 216 \mu \mathrm{mol}, 0.2 \mathrm{eq})$ was added. After 10 minutes (2,2-dimethyl-6methylene-6H-[1,3]dioxin-4-yloxy)-trimethyl-silane (231 mg, $1.08 \mathrm{mmol}, 1 \mathrm{eq})$ dissolved in $\mathrm{CH}_{2} \mathrm{Cl}_{2}(1.5 \mathrm{~mL})$ was added dropwise. The reaction mixture was then stirred overnight reaching $\mathrm{RT}$ gradually. $\mathrm{CH}_{2} \mathrm{Cl}_{2}$ was removed under reduced pressure and the residue was taken up with AcOEt. It was washed $3 x$ with citric acid (10 \%) and 3x with satd. $\mathrm{NaHCO}_{3}$. The organic phase was dried over $\mathrm{Na}_{2} \mathrm{SO}_{4}$, filtered and evaporated under reduced pressure. FC (AcOEt/hexane 3:7) gave the title compound (155 mg, $0.32 \mathrm{mmol}, 30 \%)$. White solid. $\mathrm{M}_{\mathrm{p}}=61-63^{\circ} \mathrm{C} . \mathrm{R}_{\mathrm{f}}=0.2($ AcOEt $/$ hexane $3: 7) \cdot[\alpha]_{\mathrm{D}}=+5.2$ $\left(\mathrm{C}=0.2, \mathrm{CHCl}_{3}, \mathrm{~T}=27^{\circ} \mathrm{C}\right) .{ }^{1} \mathrm{H}-\mathrm{NMR}\left(\mathrm{CDCl}_{3}, 300 \mathrm{MHz}\right) \delta 0.055(\mathrm{~s}, 3 \mathrm{H}), 0.069(\mathrm{~s}, 3 \mathrm{H})$, $0.88(\mathrm{~s}, 9 \mathrm{H}), 2.34\left(\mathrm{dd}, 1 \mathrm{H}, J_{1}=5.3, J_{2}=14.95\right), 2.44\left(\mathrm{dd}, 1 \mathrm{H}, J_{1}=8.5, J_{2}=14.63\right), 3.30$ (s, 1H), 3.63-3.66 (m, 1H), $3.86(\mathrm{~d}, 2 \mathrm{H}, \mathrm{J}=3.12), 4.26-4.29(\mathrm{~m}, 1 \mathrm{H}), 5.12(\mathrm{~s}, 2 \mathrm{H}), 5.33(\mathrm{~s}$, 1H), 5.41 (br . d, $1 \mathrm{H}, J=8.7), 7.32-7.37(\mathrm{~m}, 5 \mathrm{H}) .{ }^{13} \mathrm{C}-\mathrm{NMR}\left(\mathrm{CDCl}_{3}, 75 \mathrm{MHz}\right)-5.4,18.3$, $24.9,25.5,26.0,38.7,54.3,66.1,67.3,70.1,95.5,106.8,128.3,128.5,128.8,136.5$, 156.6, 161.2, 168.6. IR 3580-3165m, 2360m, 2355m, 1728.2s. MS $502.2\left(8.6,[\mathrm{M}+\mathrm{Na}]^{+}\right)$, $444.2\left(100,[\mathrm{M} \text {-acetone+Na] }]^{+}\right)$. HRMS calcd. for $\mathrm{C}_{24} \mathrm{H}_{37} \mathrm{NO}_{7} \mathrm{SiNa}(\mathrm{M}+\mathrm{Na})^{+}:$502.2232, found: 502.2239 . 
<smiles>[Z]NC(C[OH2+])[C@@H](O)CC1=CC(=O)OC(C)(C)O1</smiles><smiles>[R5]CC(N[Z])[C@@H](O)CC(=O)CC(=O)OC</smiles>

To a solution of [1-(tert-Butyl-dimethyl-silanyloxymethyl)-3-(2,2-dimethyl-6-oxo-6H[1,3]dioxin-4-yl)-((3R)-2-hydroxy-propyl]-(2R)carbamic acid benzyl ester (28.0 mg, 58.4 $\mu \mathrm{mol})$ in Toluene $(0.5 \mathrm{~mL})$ was added $\mathrm{MeOH}(9.5 \mathrm{~mL}, 234 \mu \mathrm{mol}, 4 \mathrm{eq})$ and the reaction mixture was refluxed for 4 hours. Then it was cooled until RT and the solvents were removed under reduced pressure. FC (AcOEt/hexane 3:7) gace the title compound (23.2 mg, $48.5 \mu \mathrm{mol}, 83 \%) . \mathrm{R}_{\mathrm{f}}=0.23\left(\right.$ AcOEt/hexane 3:7). $[\alpha]_{\mathrm{D}}=-1.34(\mathrm{C}=0.75$, $\left.\mathrm{CHCl}_{3}, \mathrm{~T}=24.9^{\circ} \mathrm{C}\right) .{ }^{1} \mathrm{H}-\mathrm{NMR}\left(\mathrm{CDCl}_{3}, 300 \mathrm{MHz}\right) \delta 0.054(\mathrm{~s}, 3 \mathrm{H}), 0.063(\mathrm{~s}, 3 \mathrm{H}), 0.88(\mathrm{~s}$, $9 \mathrm{H}), 2.65\left(\mathrm{dd}, 1 \mathrm{H}, J_{1}=4.36, J_{2}=17.43\right), 2.79\left(\mathrm{dd}, 1 \mathrm{H}, J_{1}=8.4, J_{2}=17.43\right), 3.35(\mathrm{~s}, 1 \mathrm{H})$, $3.48(\mathrm{~s}, 2 \mathrm{H}), 3.63-3.65(\mathrm{~m}, 1 \mathrm{H}), 3.72(\mathrm{~s}, 3 \mathrm{H}), 3.81(\mathrm{~d}, 2 \mathrm{H}, \mathrm{J}=4.35), 4.44-4.49(\mathrm{~m}, 1 \mathrm{H})$, 5.1 (s, 2H), 5.32 (br. d, $J=9.34), 7.31-7.37$ (m, 5H). ${ }^{13} \mathrm{C}-\mathrm{NMR}\left(\mathrm{CDCl}_{3}, 75 \mathrm{MHz}\right)-5.4$, $18.3,25.9,46.8,49.7,52.5,54.3,65.0,67.0,68.0,128.0,128.1,128.5,136.2,156.3$, 167.1, 202.0. IR 3617-3213m, 1745m, 1717s. MS 476.2 (100, [M+Na] $\left.]^{+}\right), 444.2$ (79, [M$\mathrm{MeOH}+\mathrm{Na}]^{+}$. HRMS calcd. for $\mathrm{C}_{22} \mathrm{H}_{35} \mathrm{NO}_{7} \mathrm{SiNa}(\mathrm{M}+\mathrm{Na})^{+}:$476.2075, found: 476.2070 . 
(6R)-6-Benzyloxycarbonylamino-7-(tert-butyl-dimethyl-silanyloxy)-(3R,5R)-4,5-

hydroxy-3-oxo-heptanoic acid methyl ester<smiles>[Z]NC(CO[Sb])C(O)CC(=O)CC(=O)OC</smiles>

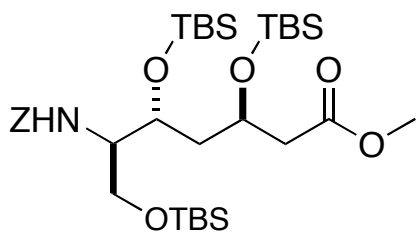

A solution of (6R)-6-Benzyloxycarbonylamino-7-(tert-butyl-dimethyl-silanyloxy)-(5R)-5hydroxy-3-oxo-heptanoic acid methyl ester $(0.23 \mathrm{~g}, 0.51 \mathrm{mmol})$ in $\mathrm{CH}_{3} \mathrm{CN}(2 \mathrm{~mL})$ was cooled to $-35{ }^{\circ} \mathrm{C}$ and $\mathrm{Me}_{4} \mathrm{~N}(\mathrm{OAc})_{3} \mathrm{BH}$ (939 mg, $3.57 \mathrm{mmol}, 7$ eq) dissolved in $\mathrm{CH}_{3} \mathrm{CN} / \mathrm{AcOH}(1.5 \mathrm{~mL} / 1.5 \mathrm{~mL})$ was added. The reaction mixture was stirred at this temperature for 62 hours. It was then allowed to reach $0{ }^{\circ} \mathrm{C}$ and a saturated solution of Na-K-tartrate was added. The solution was stirred at this temperature for 4 hours. The phases were separated and the aqueous phase extracted $3 x$ with AcOEt. The combined organic layers were washed with brine, dried over $\mathrm{Na}_{2} \mathrm{SO}_{4}$, filtered and evaporated under reduced pressure. The NMR spectrum showed a pure compound which was directly introduced in the next step.

This compound $(0.51 \mathrm{mmol})$ was dissolved in dry $\mathrm{CH}_{2} \mathrm{Cl}_{2}(0.4 \mathrm{~mL})$ and cooled to -20 ${ }^{\circ} \mathrm{C} .2,6$ lutidine $(238 \mu \mathrm{l}, 219 \mu \mathrm{mol}, 4 \mathrm{eq})$ was added dropwise followed by the dropwise addition of TBSOTf (351 $\mu \mathrm{l}, 404 \mu \mathrm{mol}, 3 \mathrm{eq})$. The reaction mixture was stirred at $-20{ }^{\circ} \mathrm{C}$ for 1h30. It was diluted with $\mathrm{CH}_{2} \mathrm{Cl}_{2}(3 \mathrm{~mL})$, washed $3 \mathrm{x}$ with citric acid (10\%) and 2x with $\mathrm{H}_{2} \mathrm{O}$. It was dried over $\mathrm{MgSO}_{4}$, filtered and evaporated under reduced pressure. FC (AcOEt/Hexane 5:95) gave the title compound $(240.0 \mathrm{mg}, 0.35 \mathrm{mmol}, 69 \%(2$ steps)). $\mathrm{R}_{\mathrm{f}}=0.5$ (AcOEt/Hexane 1:9). ${ }^{1} \mathrm{H}-\mathrm{NMR}\left(\mathrm{CDCl}_{3}, 300 \mathrm{MHz}\right) \delta 0.023(\mathrm{~s}, 3 \mathrm{H}), 0.033$ (s, 3H), $0.054(\mathrm{~s}, 3 \mathrm{H}), 0.077(\mathrm{~s}, 3 \mathrm{H}), 0.087(\mathrm{~s}, 3 \mathrm{H}), 0.102(\mathrm{~s}, 3 \mathrm{H}), 0.84(\mathrm{~s}, 9 \mathrm{H}), 0.88$ (s, $18 \mathrm{H}), 1.57-1.69(\mathrm{~m}, 1 \mathrm{H}), 1.79-1.91\left(\mathrm{ddd}, 1 \mathrm{H}, J_{1}=4.67, J_{2}=9.03, J_{3}=13.69\right), 2.37$ (dd, $\left.1 \mathrm{H}, J_{1}=8.09, J_{2}=14.31\right), 2.49\left(\mathrm{dd}, 1 \mathrm{H}, J_{1}=4.36, J_{2}=14.31\right), 3.4-3.5(\mathrm{~m}, 1 \mathrm{H}), 3.59-$ 
$3.64(\mathrm{~m}, 2 \mathrm{H}), 3.66(\mathrm{~s}, 3 \mathrm{H}), 4.04\left(\mathrm{dd}, 1 \mathrm{H}, J_{1}=4.98, J_{2}=9.33\right), 4.08-4.16(\mathrm{~m}, 1 \mathrm{H}), 5.02(\mathrm{~d}$, $1 \mathrm{H}, J=8.42), 5.1(\mathrm{~s}, 2 \mathrm{H}), 7.32-7.38(\mathrm{~m}, 5 \mathrm{H}) .{ }^{13} \mathrm{C}-\mathrm{NMR}\left(\mathrm{CDCl}_{3}, 75 \mathrm{MHz}\right)-5.3,-4.8,-4.7$, $-4.4,-4.1,18.0,18.1,25.8,25.9,26.0,42.0,42.3,51.5,54.5,61.1,66.6,66.8,128.1$, 128.2, 128.4, 136.3, 156.0, 171.2. MS 706.4 (100, $\left.[\mathrm{M}+\mathrm{Na}]^{+}\right)$. HRMS calcd. for $\mathrm{C}_{34} \mathrm{H}_{65} \mathrm{NO}_{7} \mathrm{Si}_{3} \mathrm{Na}(\mathrm{M}+\mathrm{Na})^{+}:$706.3961, found: 706.3953.

(6R)-6-Benzyloxycarbonylamino-7-(tert-butyl-dimethyl-silanyloxy)-(3S,5R)-4,5hydroxy-3-oxo-heptanoic acid methyl ester<smiles>[Z]NC(C[OH2+])[C@@H](O)CC(=O)CC(=O)OC</smiles><smiles>[Z]NC(C[OH2+])[C@@H](O)C[C@@H](O)CC(=O)OC</smiles>

A solution of $\mathrm{Et}_{3} \mathrm{~B}(330 \mu \mathrm{l}, 330 \mu \mathrm{mol}, 1.5 \mathrm{eq}, 1 \mathrm{M}$ sol. in hexane), pivalic acid (1.1 mg, 11 $\mu \mathrm{mol}, 0.05 \mathrm{eq})$ and $\mathrm{MeOH}(330 \mu \mathrm{l})$ was stirred at RT fort 1 hour. Then it was cooled to $-70{ }^{\circ} \mathrm{C}$ (6R)-6-Benzyloxycarbonylamino-7-(tert-butyl-dimethyl-silanyloxy)-(5R)-5hydroxy-3-oxo-heptanoic acid methyl ester $(0.10 \mathrm{~g}, 0.22 \mathrm{mmol})$ in dry THF $(500 \mu \mathrm{l})$ was added dropwise followed by $\mathrm{NaBH}_{4}(33 \mathrm{mg}, 0.88 \mathrm{mmol}, 4 \mathrm{eq})$. The reaction mixture was stirred at $-70{ }^{\circ} \mathrm{C}$ for 4 hours. $\mathrm{H}_{2} \mathrm{O}_{2}(1 \mathrm{~mL})$ and $\mathrm{H}_{2} \mathrm{O}(1.5 \mathrm{~mL})$ were then added at -70 ${ }^{\circ} \mathrm{C}$ and the reaction allowed to warm until RT. Then $\mathrm{CHCl}_{3}$ and $\mathrm{H}_{2} \mathrm{O}$ were added and the aqueous phase extracted with chloroform. The combined organic layers were washed with $5 \times \mathrm{H}_{2} \mathrm{O}$, dried over $\mathrm{Na}_{2} \mathrm{SO}_{4}$, filtered and evaporated under reduced pressure. FC (Hexane/AcOET 6:4) gave the cis title compound (70.0 mg, $15.4 \mu \mathrm{mol}$, 69\%). $\mathrm{R}_{\mathrm{f}}=0.18(\mathrm{AcOEt} / \mathrm{Hexane} 4: 6) .[\alpha]_{\mathrm{D}}=-11.68\left(\mathrm{C}=2.22, \mathrm{CHCl}_{3}, \mathrm{~T}=31.2{ }^{\circ} \mathrm{C}\right) .{ }^{1} \mathrm{H}-$ $\operatorname{NMR}\left(\mathrm{CDCl}_{3}, 300 \mathrm{MHz}\right) \delta 0.052(\mathrm{~s}, 3 \mathrm{H}), 0.061(\mathrm{~s}, 3 \mathrm{H}), 0.88(\mathrm{~s}, 9 \mathrm{H}), 1.5-1.6(\mathrm{~m}, 1 \mathrm{H}), 1.67-$ $1.81(\mathrm{~m}, 1 \mathrm{H}), 2.44-2.51(\mathrm{~m}, 2 \mathrm{H}), 3.56-3.65(\mathrm{~m}, 1 \mathrm{H}), 3.69(\mathrm{~s}, 3 \mathrm{H}), 3.75-3.82(\mathrm{~m}, 2 \mathrm{H}), 3.89-$ $3.93(\mathrm{~s}, 1 \mathrm{H}), 4.19-4.35(\mathrm{~m}, 2 \mathrm{H}), 5.1(\mathrm{~d}, 1 \mathrm{H}, \mathrm{J}=1.87), 5.39$ (br. d, $1 \mathrm{H}, J=9.02), 7.3-7.36$ 
(m, 5H). ${ }^{13} \mathrm{C}-\mathrm{NMR}\left(\mathrm{CDCl}_{3}, 75 \mathrm{MHz}\right)-5.4,18.2,25.9,39.4,41.5,51.8,55.0,65.0,66.8$, $68.1,71.8,127.95,128.0,128.4,136.3,156.3,172.5$. IR 3664-3143m, 1722s, $1508.8 m$. MS $478.2\left(100,[\mathrm{M}+\mathrm{Na}]^{+}\right)$. HRMS calcd. for $\mathrm{C}_{22} \mathrm{H}_{37} \mathrm{NO}_{7} \mathrm{SiNa}(\mathrm{M}+\mathrm{Na})^{+}: 478.2232$, found: 478.2225 .

(6R)-6-Benzyloxycarbonylamino-7-(tert-butyl-dimethyl-silanyloxy)-(3S,5R)-4,5hydroxy-3-oxo-heptanoic acid methyl ester<smiles>CNC(CO)C(O)CC(O)CC(=O)OC</smiles>

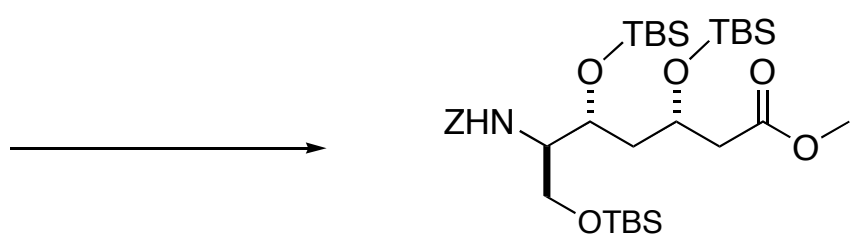

A solution of (6R)-6-Benzyloxycarbonylamino-7-(tert-butyl-dimethyl-silanyloxy)(3S,5R)-4,5-hydroxy-3-oxo-heptanoic acid methyl ester (70.0 mg, $0.15 \mathrm{mmol})$ in dry $\mathrm{CH}_{2} \mathrm{Cl}_{2}(0.5 \mathrm{~mL})$ was cooled to $-20^{\circ} \mathrm{C}$ and 2,6 lutidine $(70 \mu \mathrm{l}, 0.6 \mathrm{mmol}, 4 \mathrm{eq})$ was added dropwise followed by TBSOTf (103 $\mu \mathrm{l}, 0.45 \mathrm{mmol}, 3 \mathrm{eq})$. The reaction mixture was stirred at $-20{ }^{\circ} \mathrm{C}$ for $2 \mathrm{~h}$. Then $\mathrm{CH}_{2} \mathrm{Cl}_{2}$ was added and the organic phase was washed $3 x$ with citric acid (10\%) and $2 x$ with $\mathrm{H}_{2} \mathrm{O}$, dried over $\mathrm{Na}_{2} \mathrm{SO}_{4}$, filtered and evaporated under reduced pressure. FC (AcOEt/hexane 1:9) gave the title compound (78.0 mg, $0.11 \mathrm{mmol}, 74 \%) . R_{\mathrm{f}}=0.68\left(\right.$ AcOEt/hexane 1:4). $[\alpha]_{\mathrm{D}}=+0.64(\mathrm{C}=3.85$, $\left.\mathrm{CHCl}_{3}, \mathrm{~T}=28.1{ }^{\circ} \mathrm{C}\right) .{ }^{1} \mathrm{H}-\mathrm{NMR}\left(\mathrm{CDCl}_{3}, 300 \mathrm{MHz}\right) \delta 0.063\left(2^{*} \mathrm{~s}, 9 \mathrm{H}\right), 0.086(\mathrm{~s}, 6 \mathrm{H}), 0.103$ (s, 3H), $0.87(\mathrm{~s}, 9 \mathrm{H}), 0.89(\mathrm{~s}, 9 \mathrm{H}), 0.90(\mathrm{~s}, 9 \mathrm{H}), 1.65-1.83(\mathrm{~m}, 2 \mathrm{H}), 2.43(\mathrm{~d}, 2 \mathrm{H}, J=5.91)$, $3.54\left(\mathrm{dd}, 1 \mathrm{H}, J_{1}=7.78, J_{2}=9.02\right), 3.6(\mathrm{~s}, 3 \mathrm{H}), 3.65-3.8(\mathrm{~m}, 2 \mathrm{H}), 4.08(\mathrm{~m}, 1 \mathrm{H}), 4.12(\mathrm{dd}$, $\left.1 \mathrm{H}, J_{1}=5.29, J_{2}=5.74\right), 5.05(\mathrm{~d}, 1 \mathrm{H}, J=8.7), 5.1(\mathrm{~s}, 2 \mathrm{H}), 7.03-7.39(\mathrm{~m}, 5 \mathrm{H}) .{ }^{13} \mathrm{C}-\mathrm{NMR}$ $\left(\mathrm{CDCl}_{3}, 75 \mathrm{MHz}\right)-5.2,-4.6,-4.1,17.9,18.1,18.5,25.8,26.0,26.1,41.9,42.9,51.4$, $54.8,61.8,66.4,66.7,67.1,128.0,128.1,128.4,136.5,156.0,171.2$. IR $2957 m$, 
$2926 m, 2884 s, 2853 m, 1725 m$. MS $706.4\left(100,[M+N a]^{+}\right)$. HRMS calcd. for $\mathrm{C}_{34} \mathrm{H}_{65} \mathrm{NO}_{7} \mathrm{Si} 3 \mathrm{Na}(\mathrm{M}+\mathrm{Na})^{+}:$706.3961, found: 706.3953.

(6R)-6-(2-benzyloxy-benzoylamino)-(3R,5R)-3,5,7-tris-(tert-butyl-dimethylsilanyloxy)-heptanoic acid methyl ester

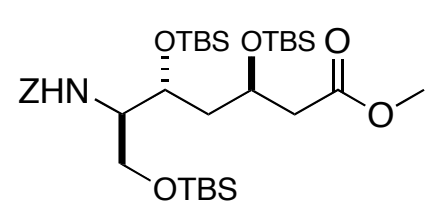

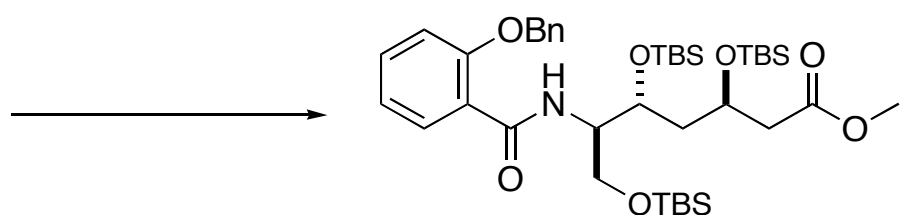

To a solution of (6R)-6-Benzyloxycarbonylamino-7-(tert-butyl-dimethyl-silanyloxy)(3R,5R)-4,5-hydroxy-3-oxo-heptanoic acid methyl ester (46 mg, 67 umol) in $\mathrm{MeOH}(1$ $\mathrm{mL}$ ) previously $3 x$ flushed with argon was added $\mathrm{Pd} / \mathrm{C}$. Then it was flushed with $\mathrm{H}_{2}$ and it was stirred at RT for 4 hours. $\mathrm{Pd} / \mathrm{C}$ was filtered over celite and the solvent was removed under reduced pressure. This product was added to a solution of $\mathrm{O}-\mathrm{Bn}$ salicylic acid (18 mg, $80 \mu \mathrm{mol}, 1.2 \mathrm{eq})$ dissolved in dry $\mathrm{CHCl}_{3}(1 \mathrm{~mL})$ with $\mathrm{EDC} . \mathrm{HCl}$ (15.3 mg, $80 \mu \mathrm{mol}, 1.2 \mathrm{eq})$, HOBt (12.2 mg, $80 \mu \mathrm{mol}, 1.2 \mathrm{eq})$ and NMM (26 $\mu \mathrm{l}, 240$ $\mu$ mol, 3 eq). The reaction mixture was stirred at RT for 20 hours. The solvent was then removed under reduced pressure and the residue taken up with AcOEt. It was washed $3 x$ with citric acid $(10 \%)$ and $3 x$ with satd. $\mathrm{NaHCO}_{3}$. The organic phase was dried over $\mathrm{MgSO}_{4}$, filtered and evaporated under reduced pressure. FC (AcOEt/hexane 1:9) gave the title compound (31.2 mg, $41 \mu \mathrm{mol}, 61 \%) . \mathrm{R}_{\mathrm{f}}=0.38\left(\right.$ AcOEt/Hexane 1:7). $[\alpha]_{D}=-26.7$ $\left(\mathrm{C}=0.81, \mathrm{CHCl}_{3}, \mathrm{~T}=24.2{ }^{\circ} \mathrm{C}\right) .{ }^{1} \mathrm{H}-\mathrm{NMR}\left(\mathrm{CDCl}_{3}, 300 \mathrm{MHz}\right) \delta-0.057(\mathrm{~s}, 3 \mathrm{H}),-0.007(\mathrm{~s}$, 3H), $0.067(\mathrm{~s}, 6 \mathrm{H}), 0.075(\mathrm{~s}, 3 \mathrm{H}), 0.107(\mathrm{~s}, 3 \mathrm{H}), 0.78(\mathrm{~s}, 9 \mathrm{H}), 0.81(\mathrm{~s}, 9 \mathrm{H}), 0.89(\mathrm{~s}, 9 \mathrm{H})$, 1.58-1.71 (m, 1H), $1.89\left(\mathrm{ddd}, J_{1}=4.67, J_{2}=9.34, J_{3}=13.7\right)$, $2.37\left(\mathrm{dd}, 1 \mathrm{H}, J_{1}=8.1, J_{2}=\right.$ 14.01), $2.52\left(\mathrm{dd}, 1 \mathrm{H}, J_{1}=4.05, J_{2}=14.01\right), 3.5(\mathrm{~m}, 1 \mathrm{H}), 3.64(\mathrm{~s}, 3 \mathrm{H}), 3.67\left(\mathrm{dd}, 1 \mathrm{H}, J_{1}=\right.$ 4.98, $\left.J_{2}=9.34\right), 4.13-4.2(\mathrm{~m}, 2 \mathrm{H}), 5.3(\mathrm{~s}, 2 \mathrm{H}), 6.9(\mathrm{~d}, 1 \mathrm{H}, J=8.4), 7.03\left(\mathrm{td}, J_{1}=0.93, J_{2}\right.$ 
$=7.78), 7.3-7.4(\mathrm{~m}, 5 \mathrm{H}), 8.0(\mathrm{~d}, 1 \mathrm{H}, J=8.72), 8.15\left(\mathrm{dd}, 1 \mathrm{H}, J_{1}=1.87, J_{2}=7.78\right) .{ }^{13} \mathrm{C}-$ NMR $\left(\mathrm{CDCl}_{3}, 75 \mathrm{MHz}\right)-5.24,-5.13,-4.85,-4.67,-4.38,17.9,18.1,25.8,25.9,42.2$, $42.3,51.4,53.4,60.6,66.6,66.8,70.8,113.2,121.3,122.4,126.6,128.0,128.6,132.3$, 136.0, 156.3, 165.0, 171.3. IR $3401 w, 1739 m, 1656 m$. MS $760\left(100,[M+H]^{+}\right)$.

(6R)-6-(2-benzyloxy-benzoylamino)-(3S,5R)-3,5,7-tris-(tert-butyl-dimethylsilanyloxy)-heptanoic acid methyl ester
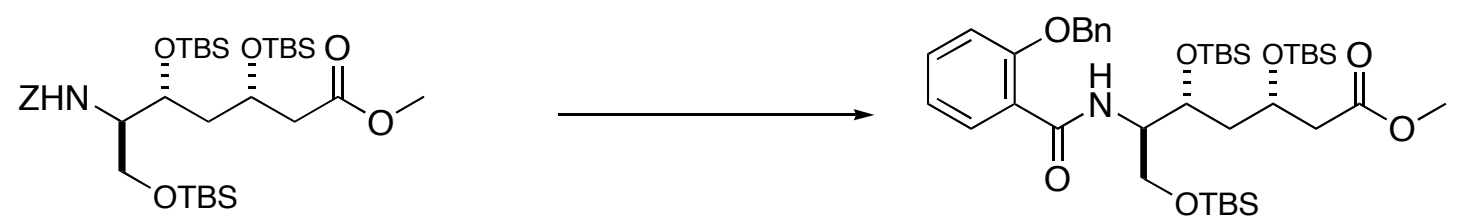

To a solution of (6R)-6-Benzyloxycarbonylamino-7-(tert-butyl-dimethyl-silanyloxy)(3S,5R)-4,5-hydroxy-3-oxo-heptanoic acid methyl ester (47 mg, 68 umol) in $\mathrm{MeOH}(1$ $\mathrm{mL}$ ) previously $3 \mathrm{x}$ flushed with argon was added $\mathrm{Pd} / \mathrm{C}$. Then it was flushed with $\mathrm{H}_{2}$ and it was stirred at RT for 4 hours. Pd/C was filtered over celite and the solvent was removed under reduced pressure. This product was added to a solution of $\mathrm{O}-\mathrm{Bn}$ salicylic acid (19 mg, $82 \mu \mathrm{mol}, 1.2 \mathrm{eq})$ dissolved in dry $\mathrm{CHCl}_{3}(1 \mathrm{~mL})$ with $\mathrm{EDC} . \mathrm{HCl}$ (15.7 $82 \mu \mathrm{mol}, 1.2 \mathrm{eq})$, HOBt (12.5 mg, $82 \mu \mathrm{mol}, 1.2 \mathrm{eq})$ and NMM $(27 \mu \mathrm{l}, 246 \mu \mathrm{mol}, 3$ eq). The reaction mixture was stirred at RT for 20 hours. The solvent was then removed under reduced pressure and the residue taken up with AcOEt. It was washed $3 x$ with citric acid (10\%) and $3 x$ with satd. $\mathrm{NaHCO}_{3}$. The organic phase was dried over $\mathrm{MgSO}_{4}$, filtered and evaporated under reduced pressure. FC (AcOEt/Hexane 1:9) gave the title compound (30 mg, $39.5 \mu \mathrm{mol}, 58 \%)$. $\mathrm{R}_{\mathrm{f}}=0.17$ (AcOEt/Hexane 1:9). Colorless oil. $[\alpha]_{D}$ $=+3.16\left(\mathrm{C}=1.45, \mathrm{CHCl}_{3}, \mathrm{~T}=28^{\circ} \mathrm{C}\right) .{ }^{1} \mathrm{H}-\mathrm{NMR}\left(\mathrm{CDCl}_{3}, 300 \mathrm{MHz}\right) \delta 0.06(\mathrm{~s}, 6 \mathrm{H}), 0.07(\mathrm{~s}$, $3 \mathrm{H}), 0.09(\mathrm{~s}, 3 \mathrm{H}), 0.094(\mathrm{~s}, 3 \mathrm{H}), 0.104(\mathrm{~s}, 3 \mathrm{H}), 0.84(\mathrm{~s}, 9 \mathrm{H}), 0.86(\mathrm{~s}, 9 \mathrm{H}), 0.89(\mathrm{~s}, 9 \mathrm{H})$, 1.68-1.83 (m, 2H), 2.34-2.48 (m, 2H), $3.49(\mathrm{~s}, 3 \mathrm{H}), 3.6\left(\mathrm{dd}, 1 \mathrm{H}, J_{1}=8.41, J_{2}=9.65\right)$, 
$1112\left(\mathrm{dd}, 1 \mathrm{H}, J_{1}=5.3, J_{2}=9.65\right), 4.13-4.22(\mathrm{~m}, 2 \mathrm{H}), 4.22-4.32(\mathrm{~m}, 1 \mathrm{H}), 5.29(\mathrm{~s}, 2 \mathrm{H})$, $6.91(\mathrm{~d}, 1 \mathrm{H}, \mathrm{J}=7.78), 7.0-7.7\left(\mathrm{td}, 2 \mathrm{H}, J_{1}=0.93, J_{2}=7.47\right), 7.28-7.38(\mathrm{~m}, 5 \mathrm{H}, 8.0(\mathrm{~d}, 1 \mathrm{H}$, $J=8.41), 8.16\left(\mathrm{dd}, 1 \mathrm{H}, J_{1}=1.87, J_{2}=7.78\right) .{ }^{13} \mathrm{C}-\mathrm{NMR}\left(\mathrm{CDCl}_{3}, 75 \mathrm{MHz}\right)-5.1,-4.5,-4.2$, $-4.0,17.9,18.1,25.9,26.0,26.1,42.1,43.2,51.4,53.8,61.2,66.5,67.1,70.8,111.2$, $121.3,122.3,126.7,128.1,128.7,132.2,132.3,135.9,156.3,165.0,171.3$. IR $3401 w$, 1739s, 1651s, 1596w, 1513m. MS $782\left(100,[\mathrm{M}+\mathrm{Na}]^{+}\right)$. HRMS calcd. for $\mathrm{C}_{40} \mathrm{H}_{69} \mathrm{NO}_{7} \mathrm{Si}_{3} \mathrm{Na}(\mathrm{M}+\mathrm{Na})^{+}:$782.4274, found: 782.4283 .

\section{References}

[1] Robertson, S. A.; Ellman, J. A.; Schultz, P. G., J. Am. Chem. Soc. 1991, 113, 27222729.

[2] Farkas, L.; Vermes, B.; Nogradi, M. Tetrahadron 1967,23,741-744.

[3] Gademann, K.; Bethuel, Y.; Angew. Chem. Int. Ed. 2004, 43, 3327-3329.

[4] Singer, R. A.; Carreira, E. M. J. Am. Chem. Soc. 1995, 117, 12360-12361.

[5] Moreau, X.; Campagne, J.-M; Tetrahedron Lett. 2001, 42, 4467-4469. 


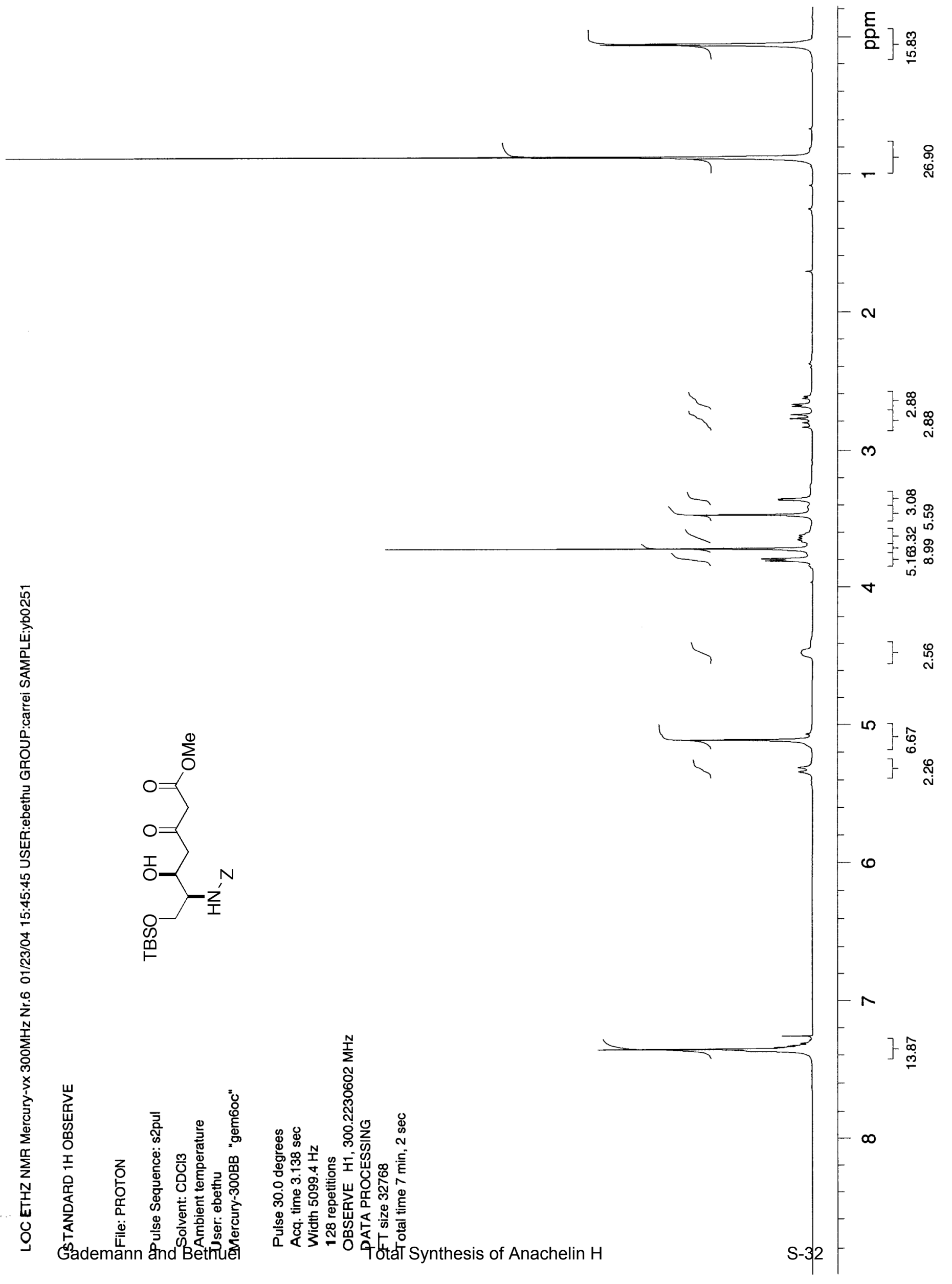




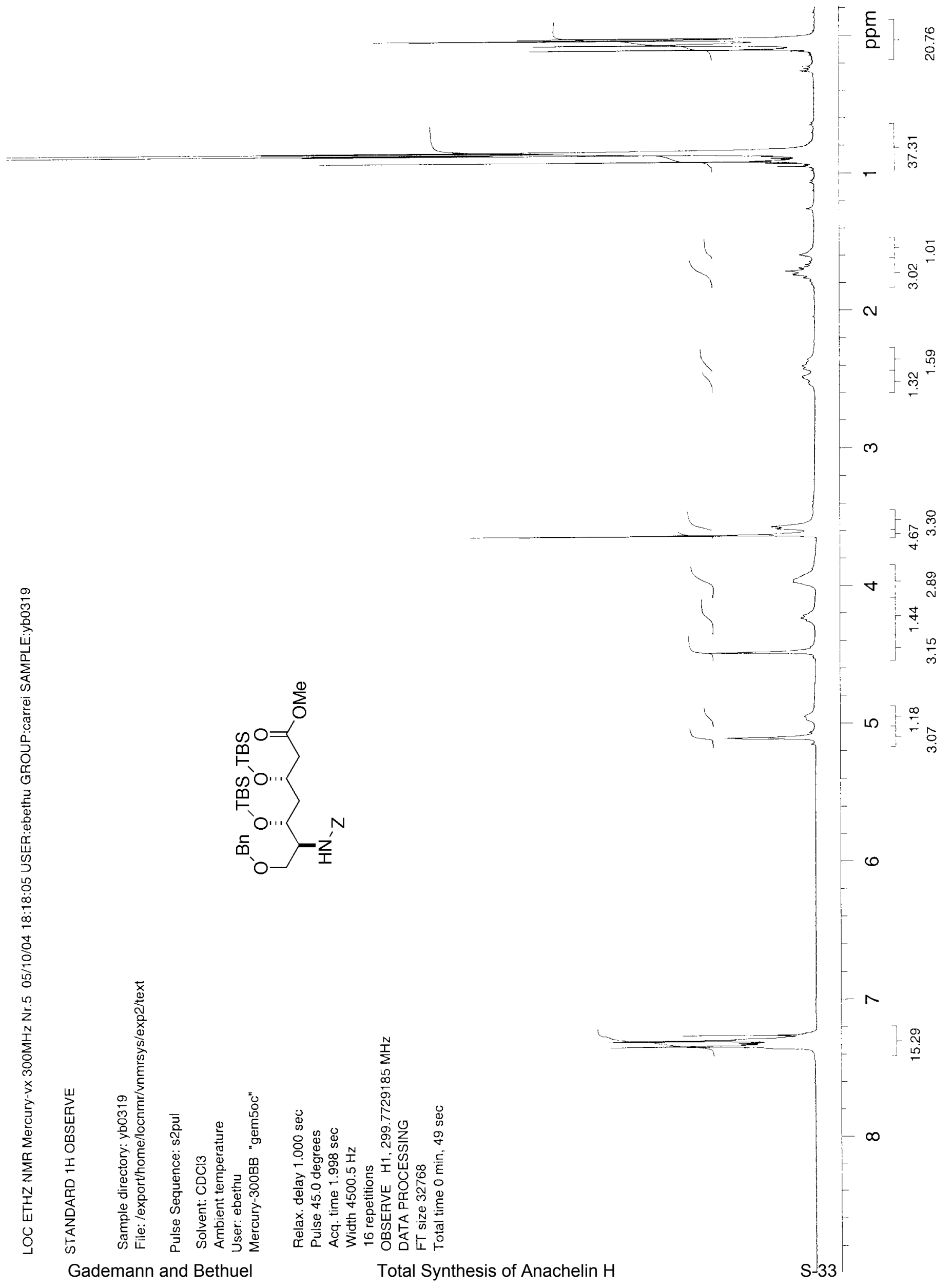




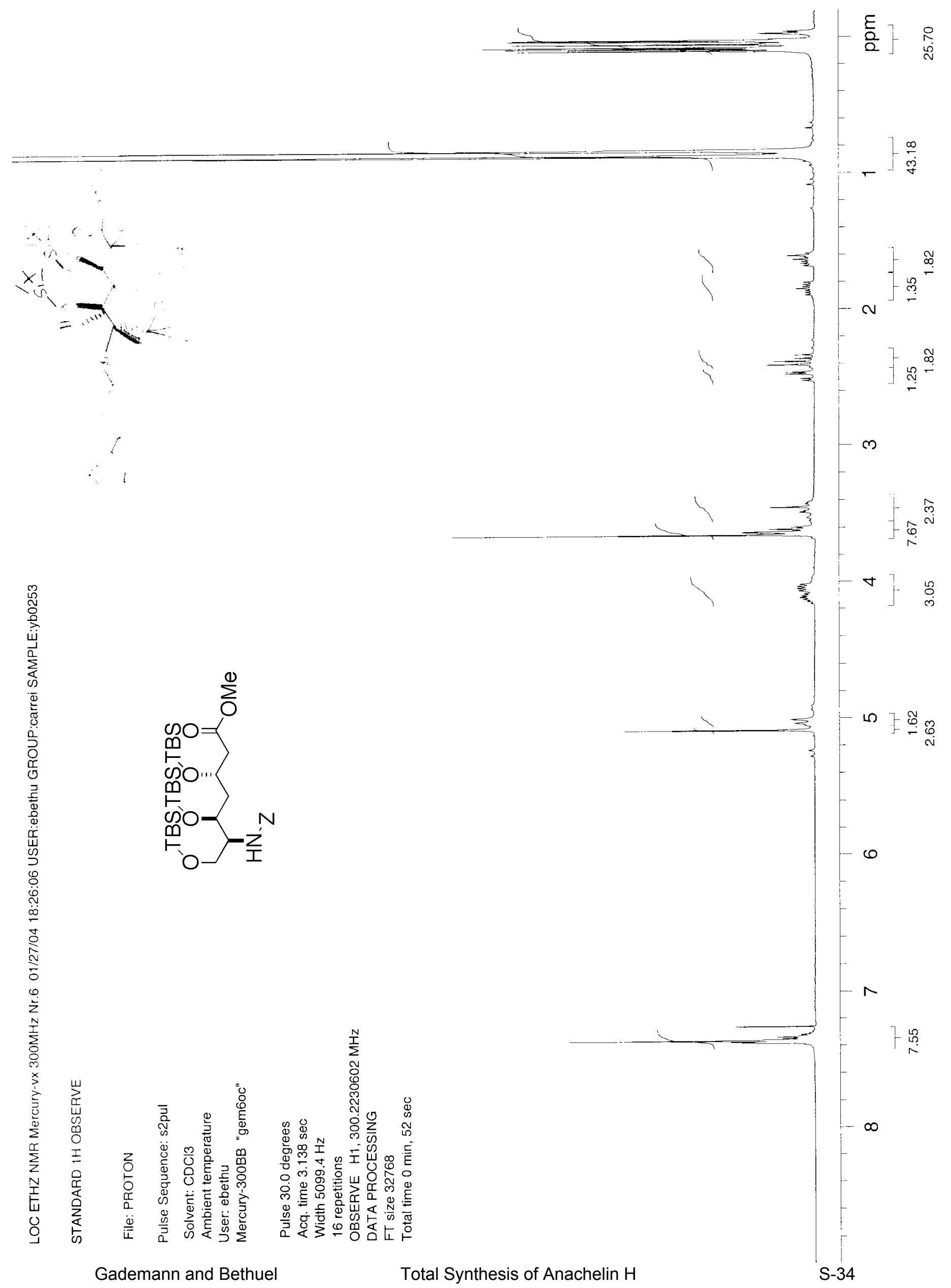




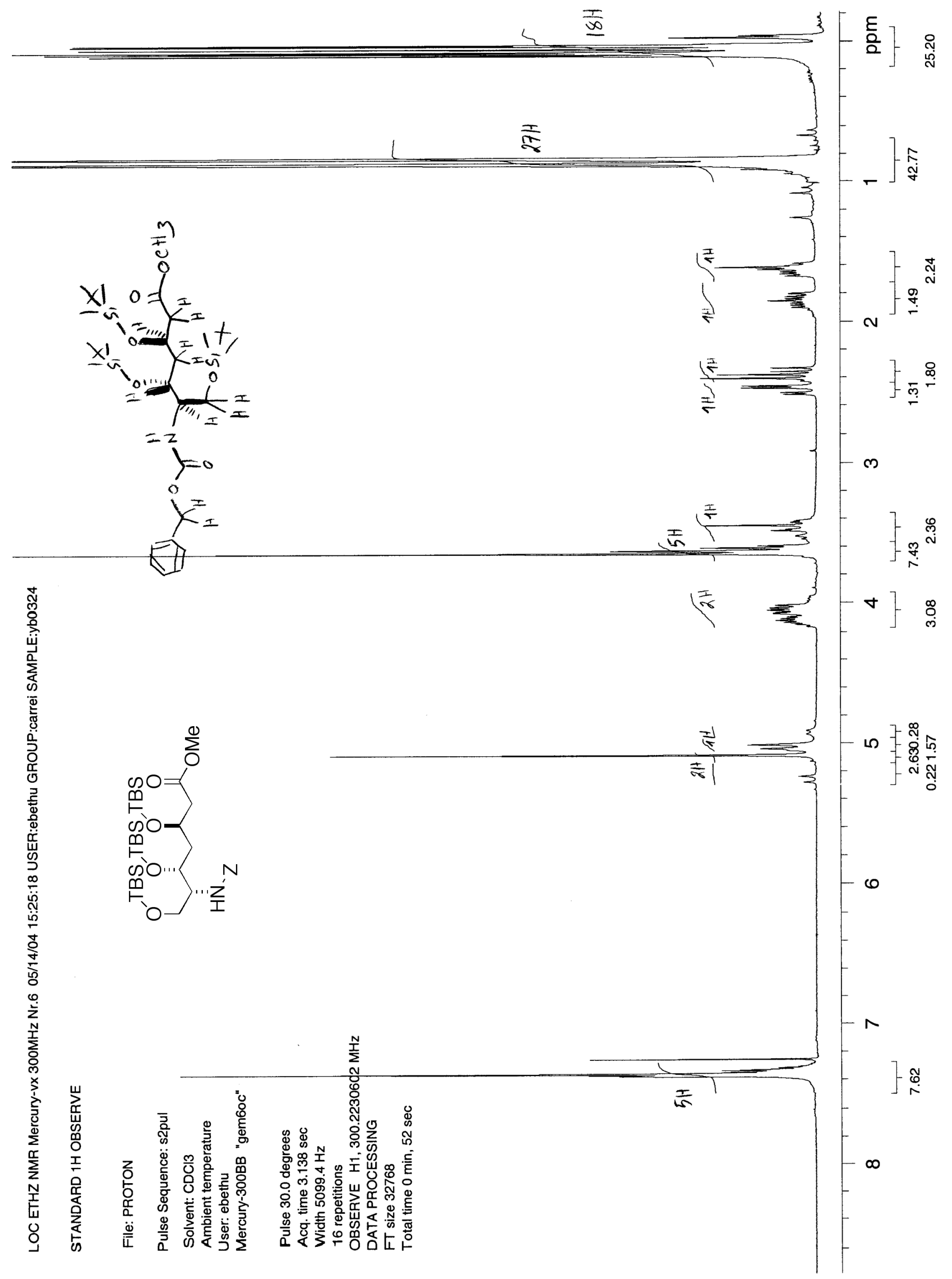




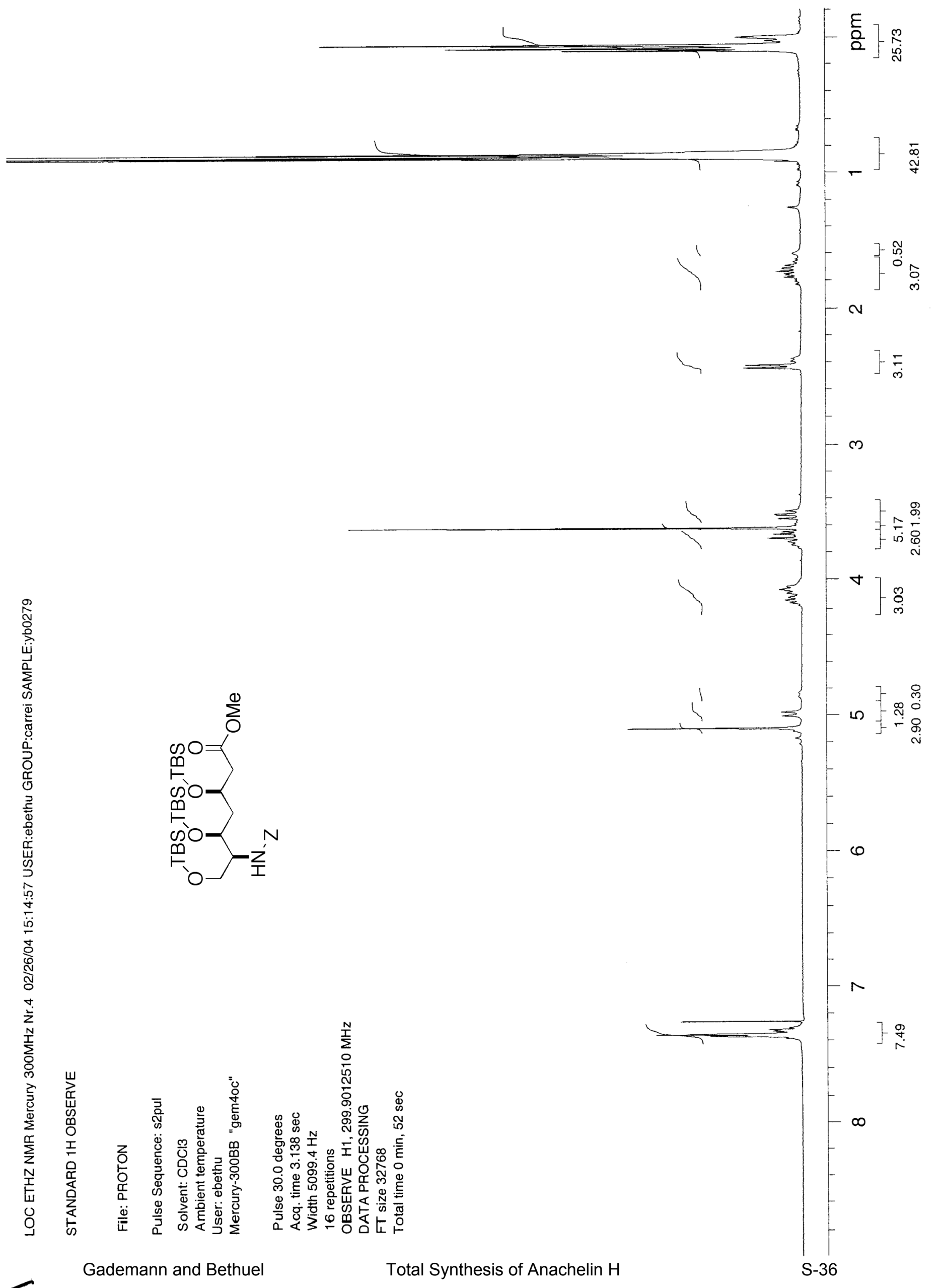




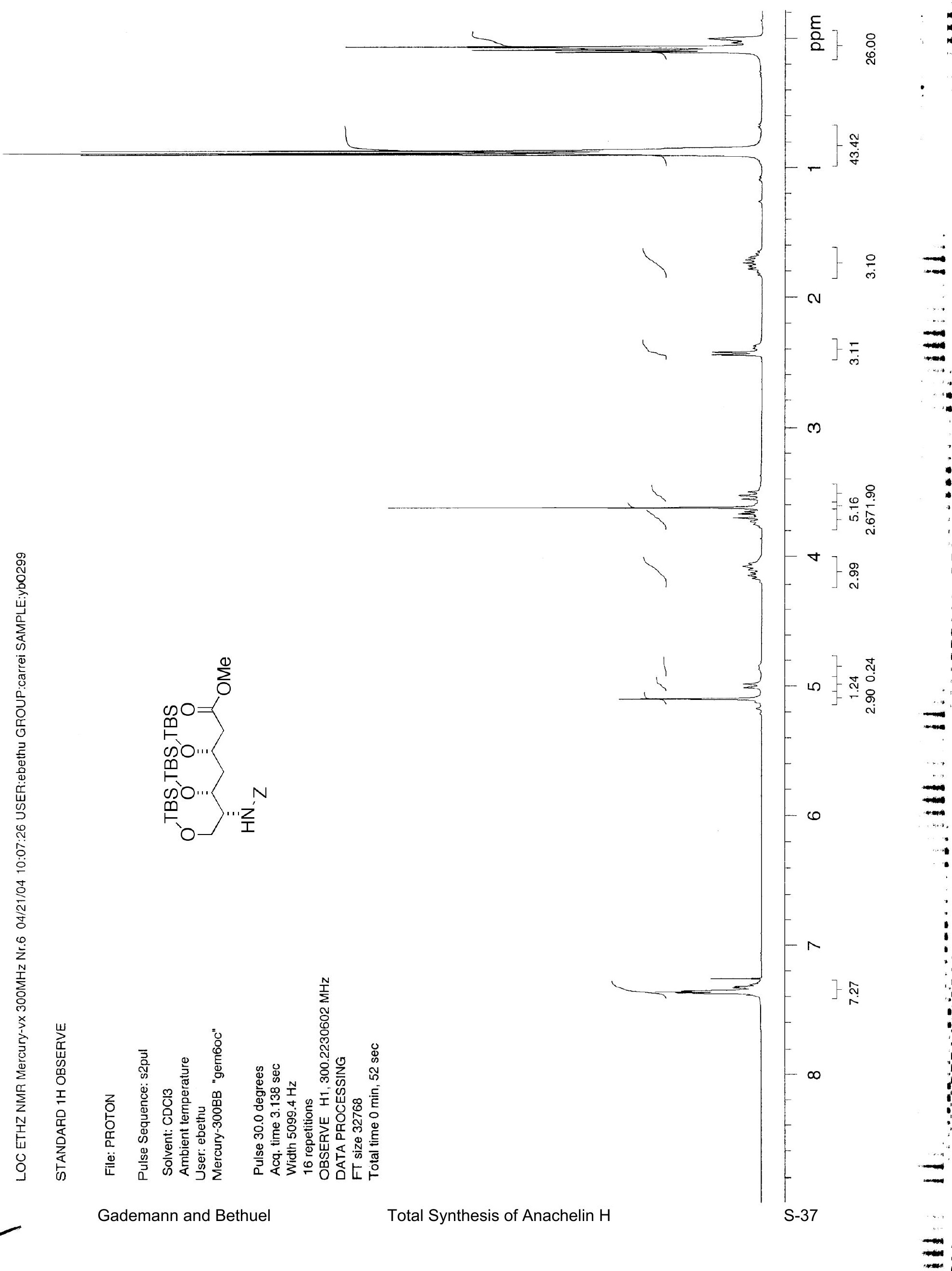




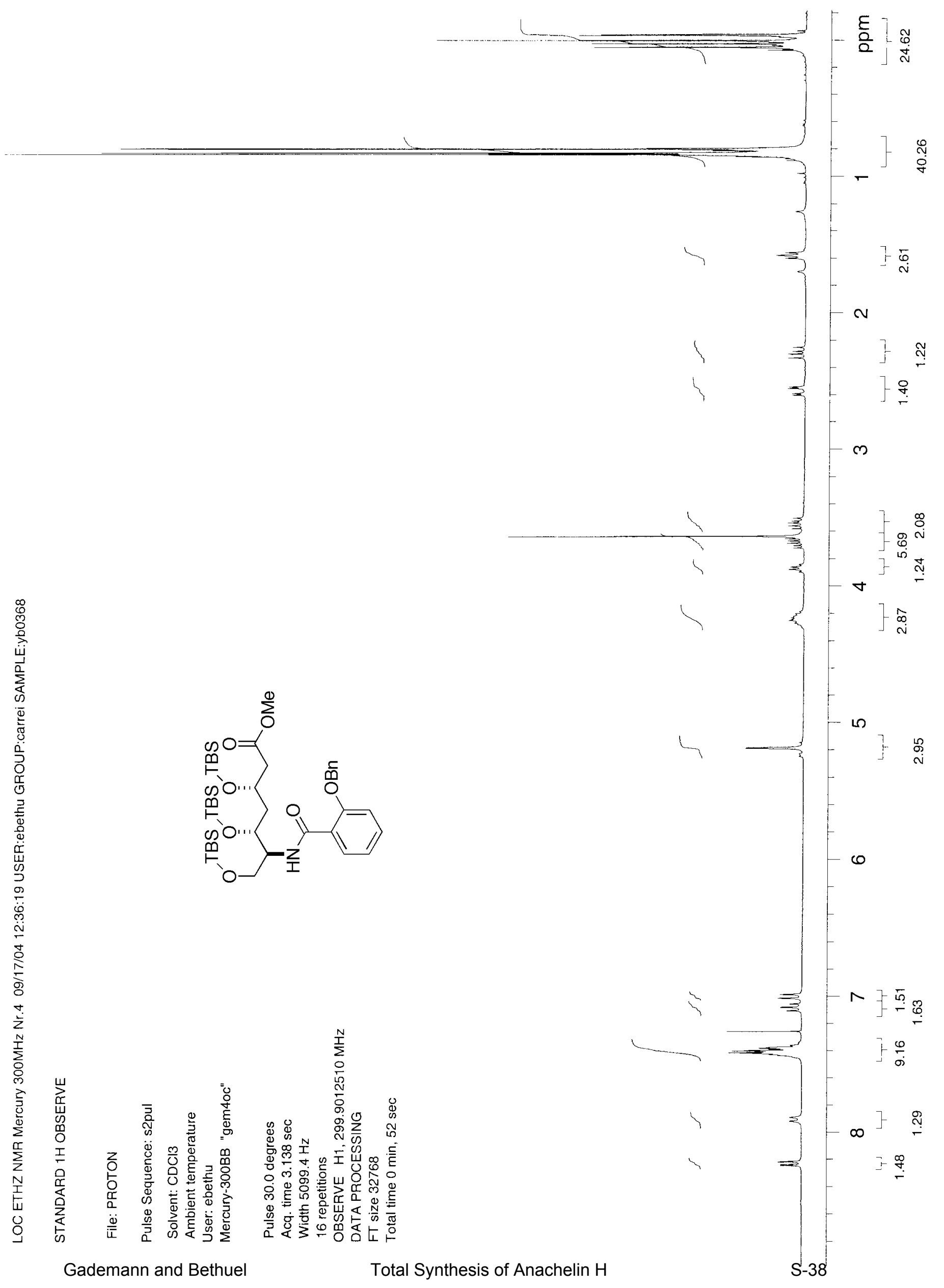




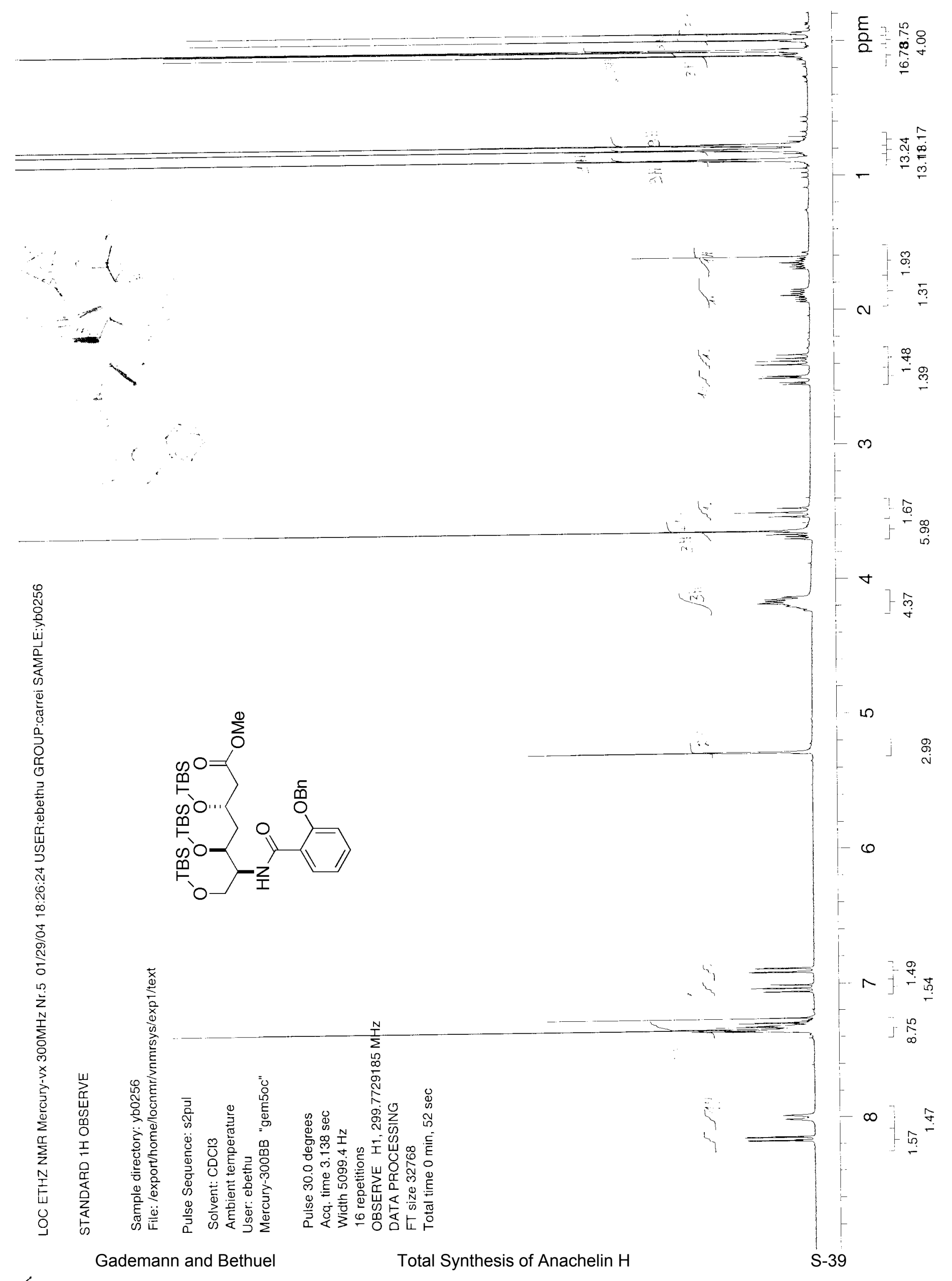




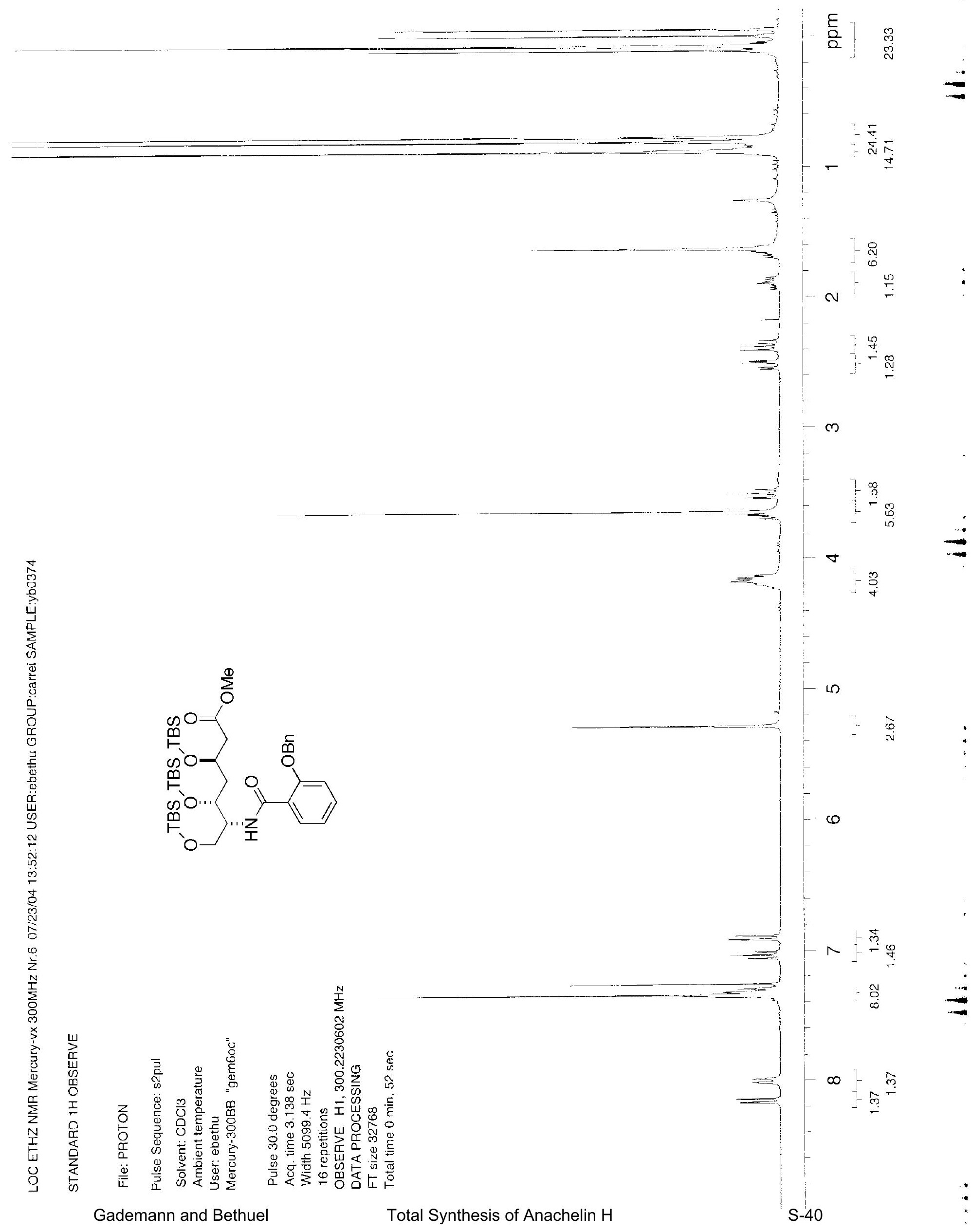




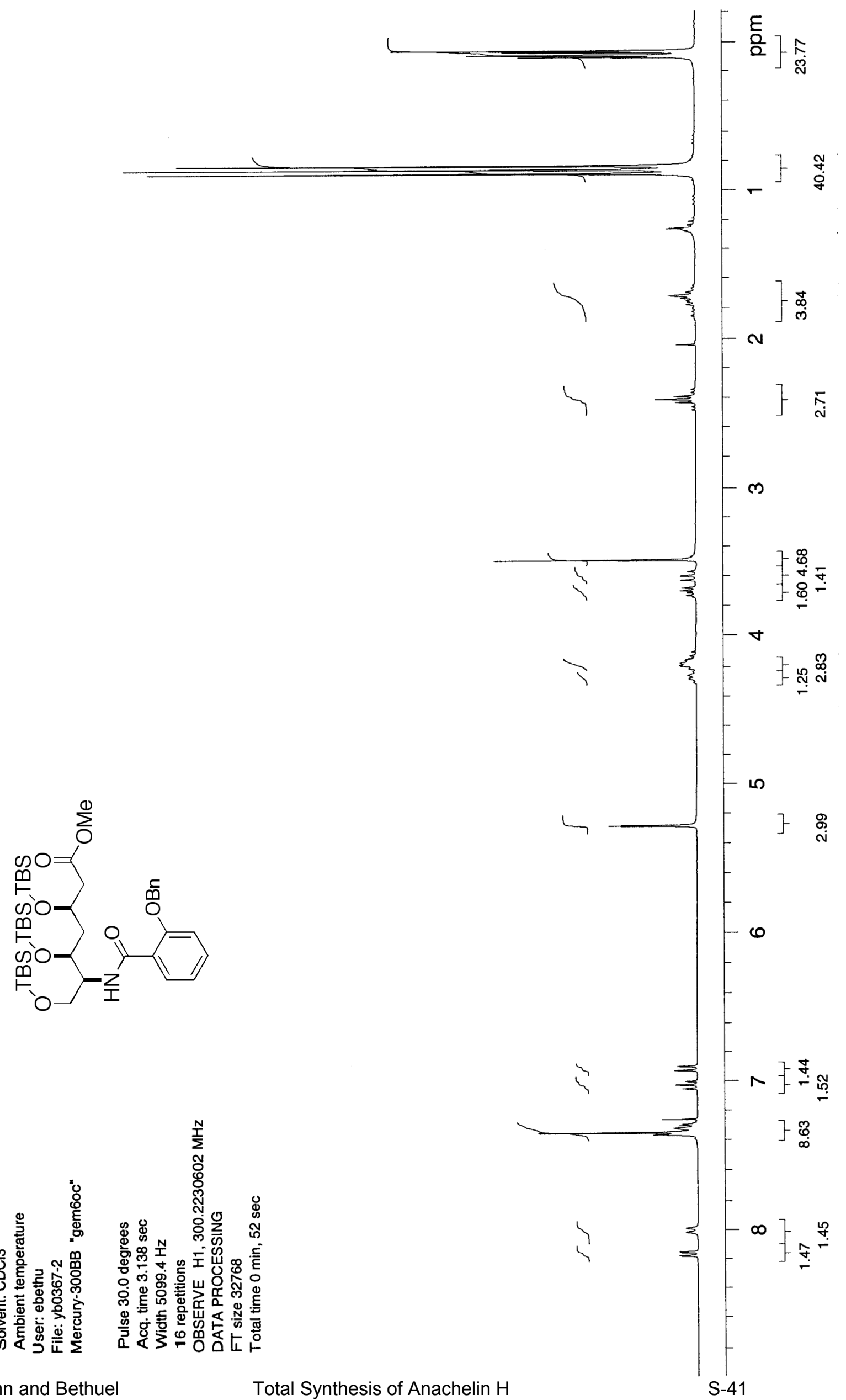




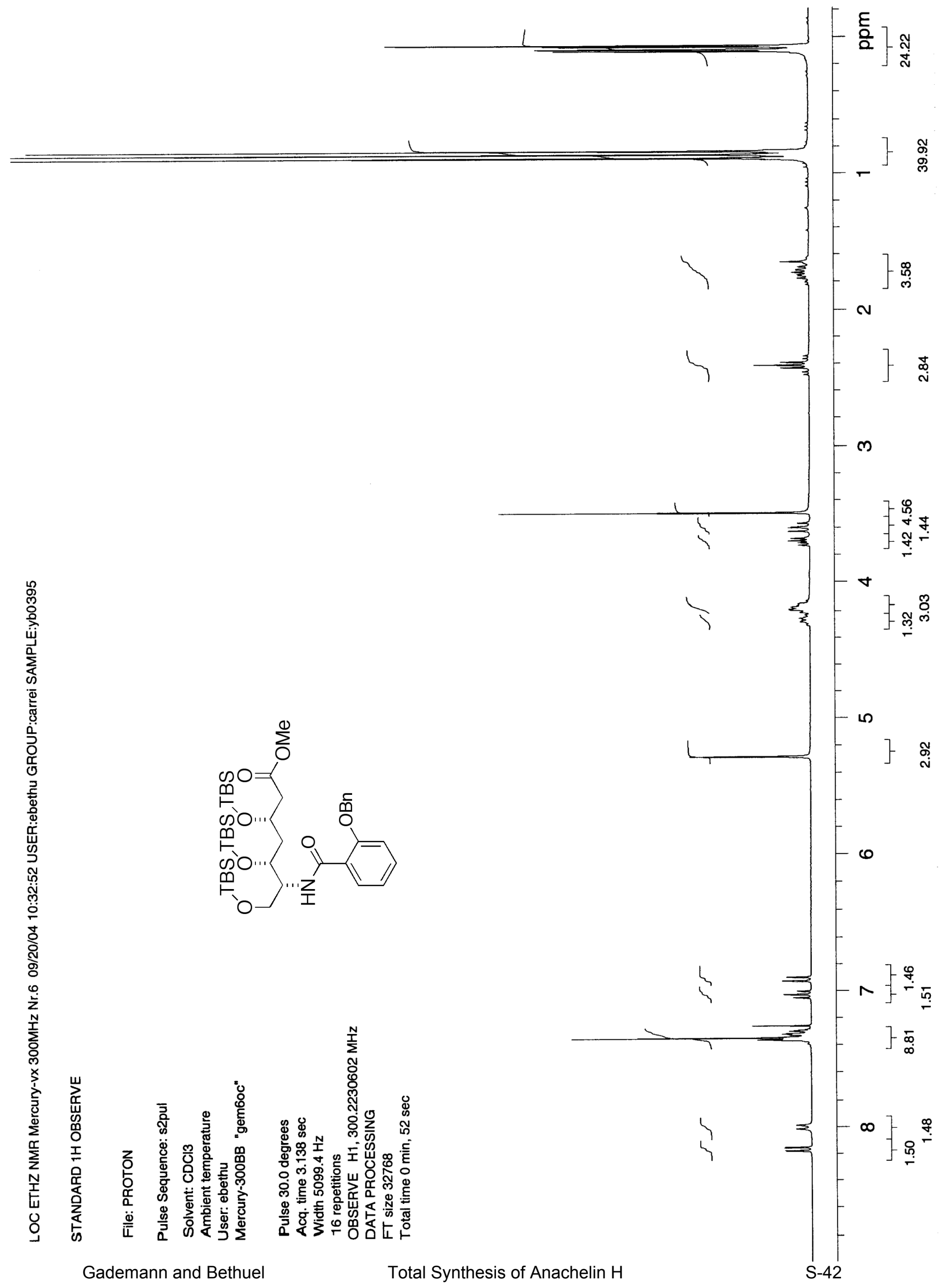




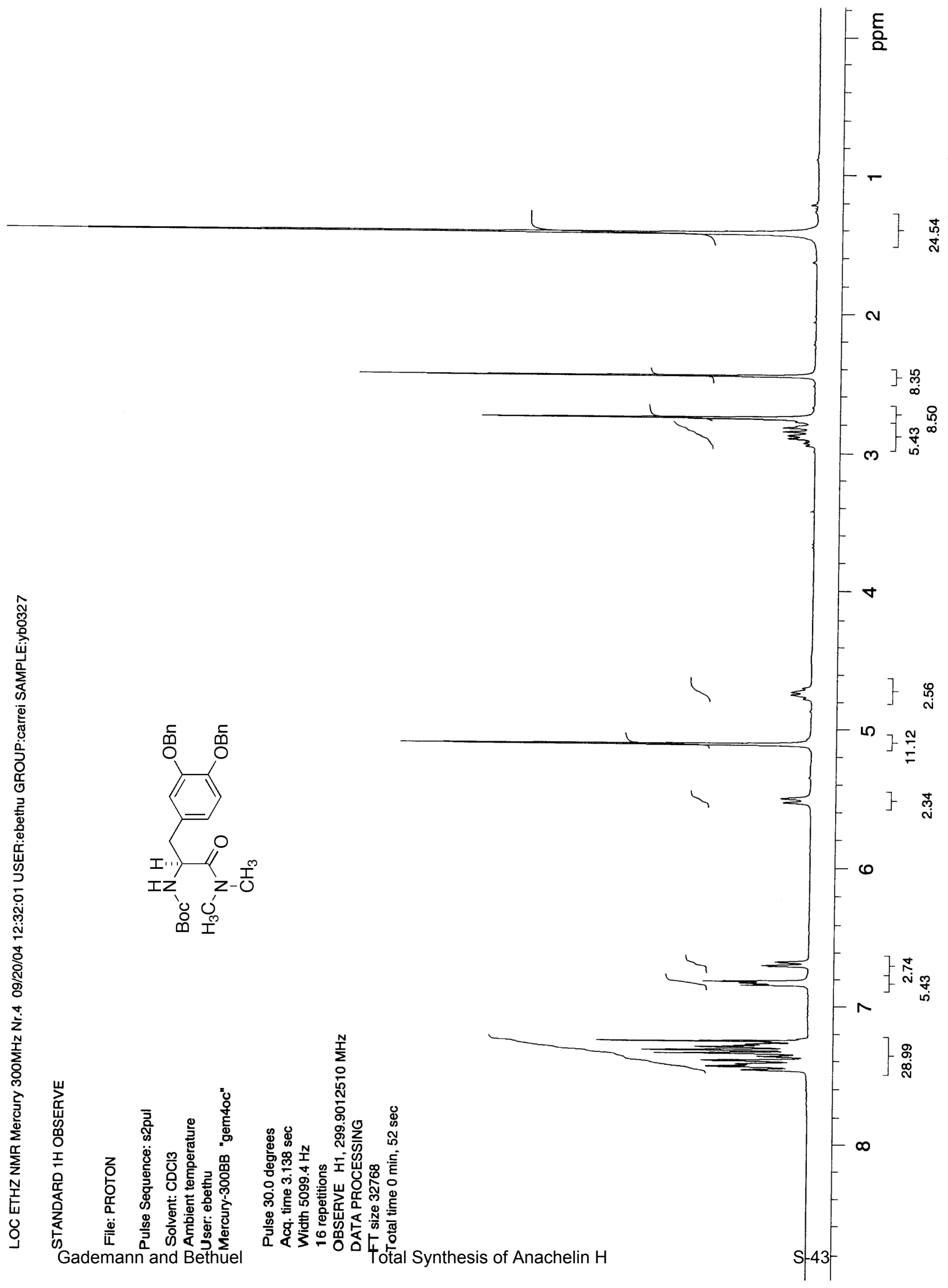




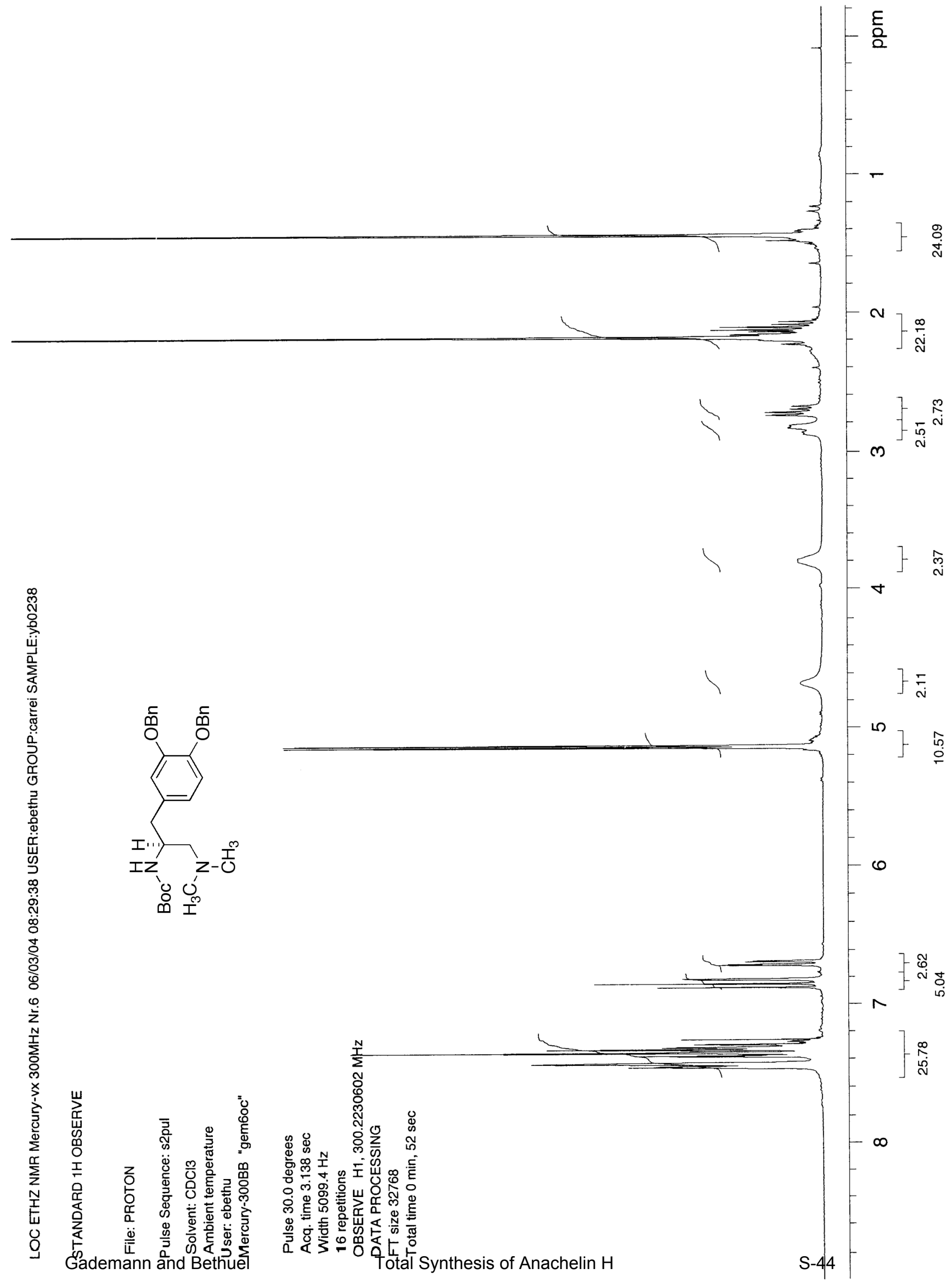




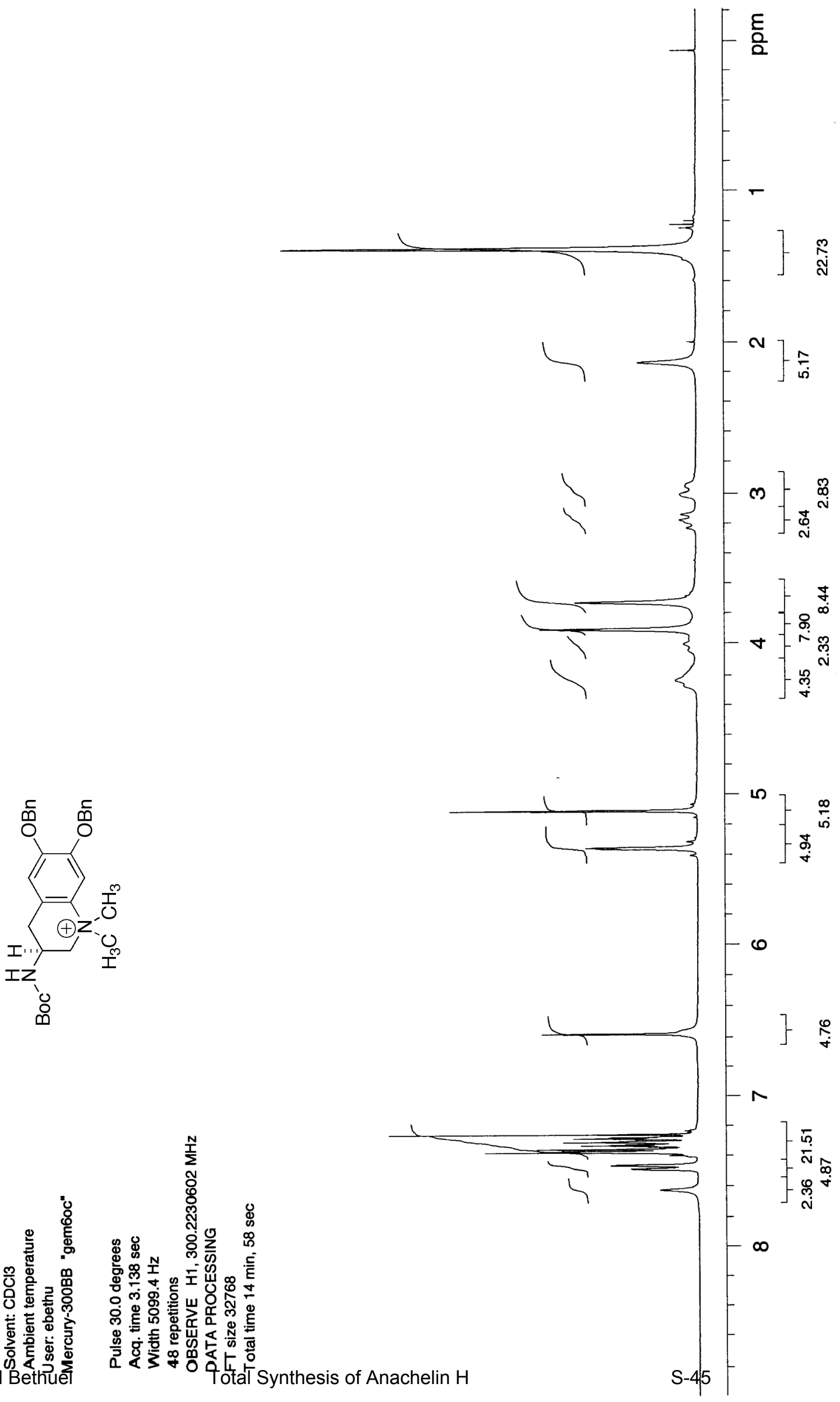



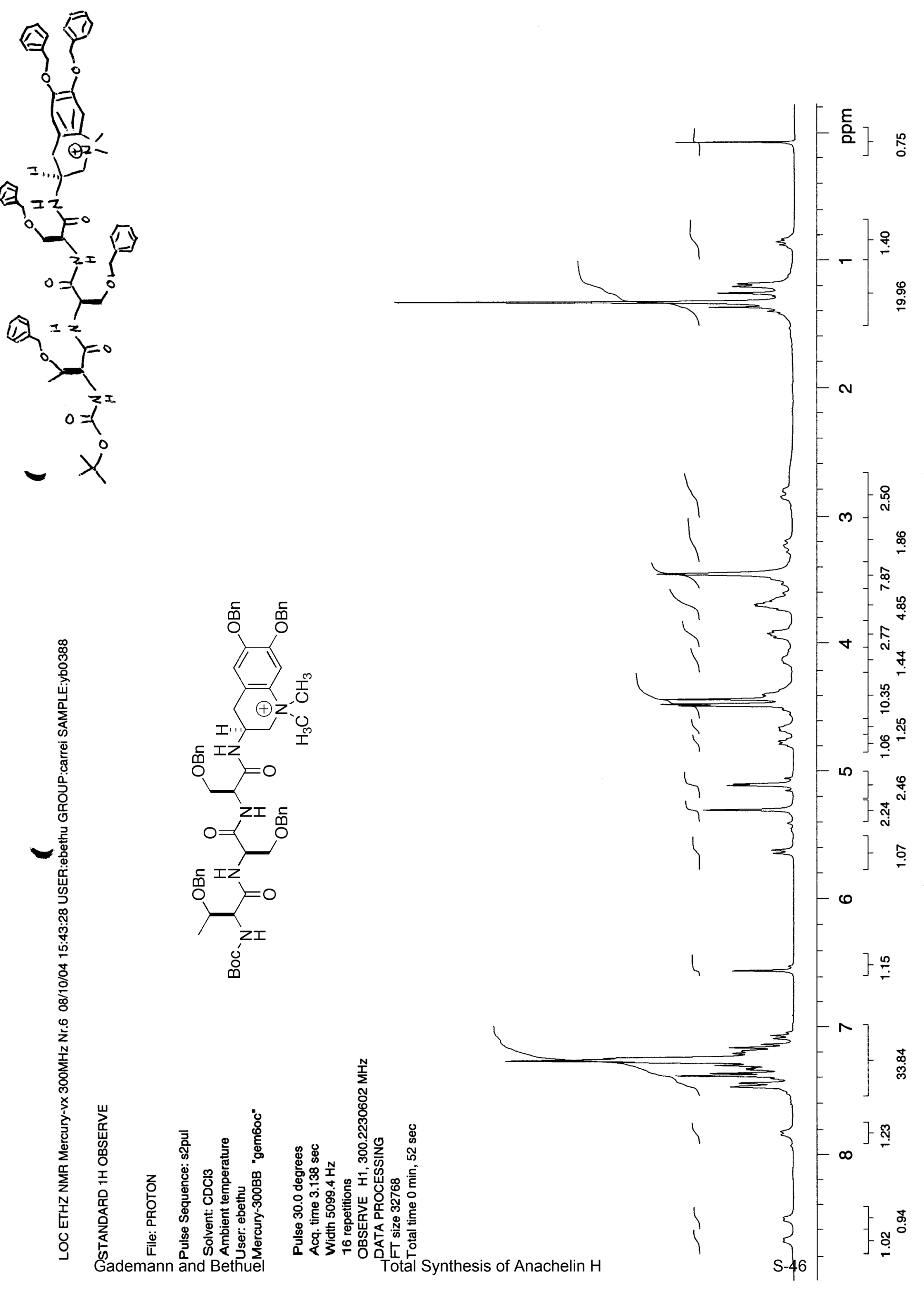


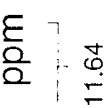
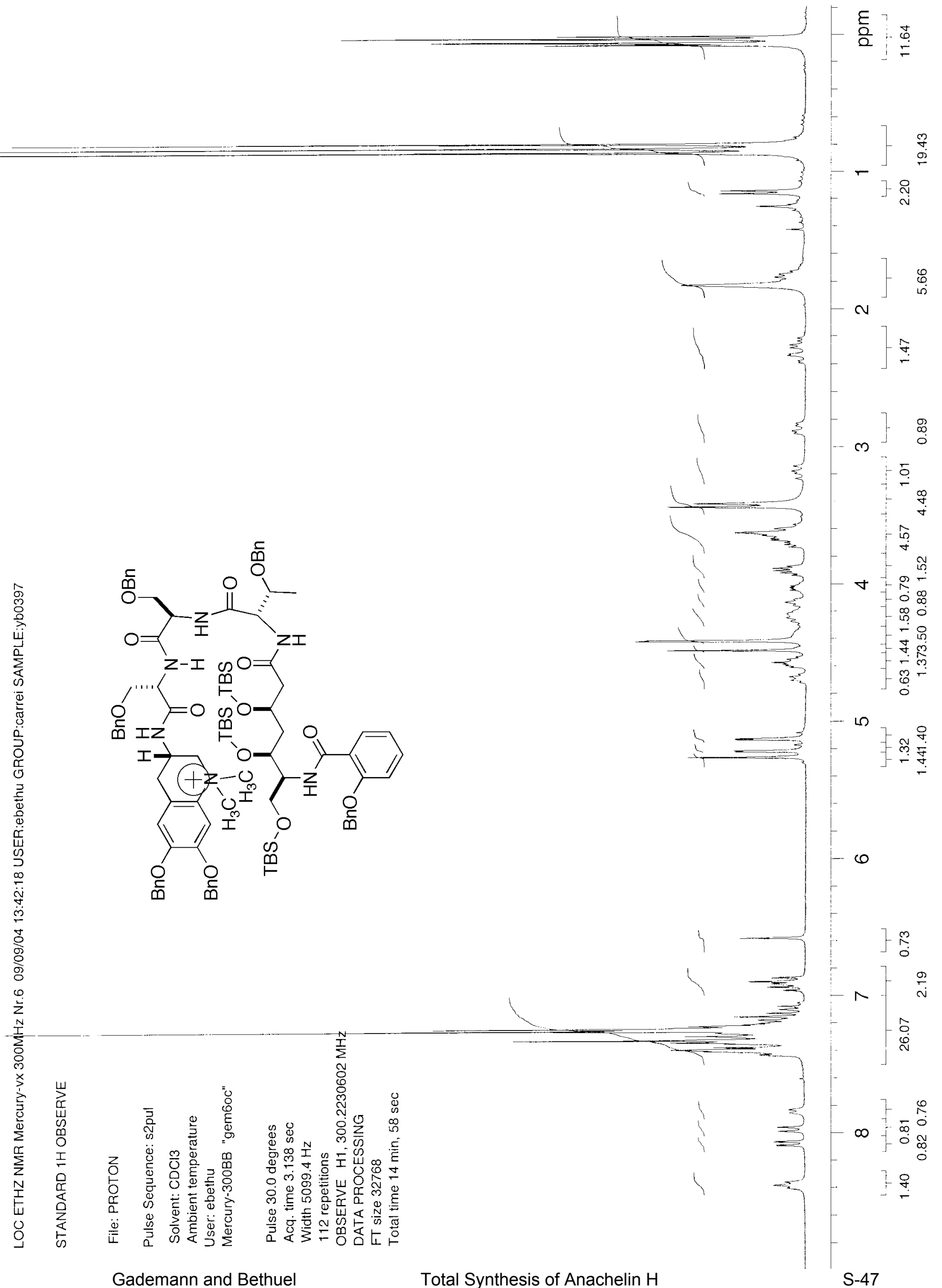


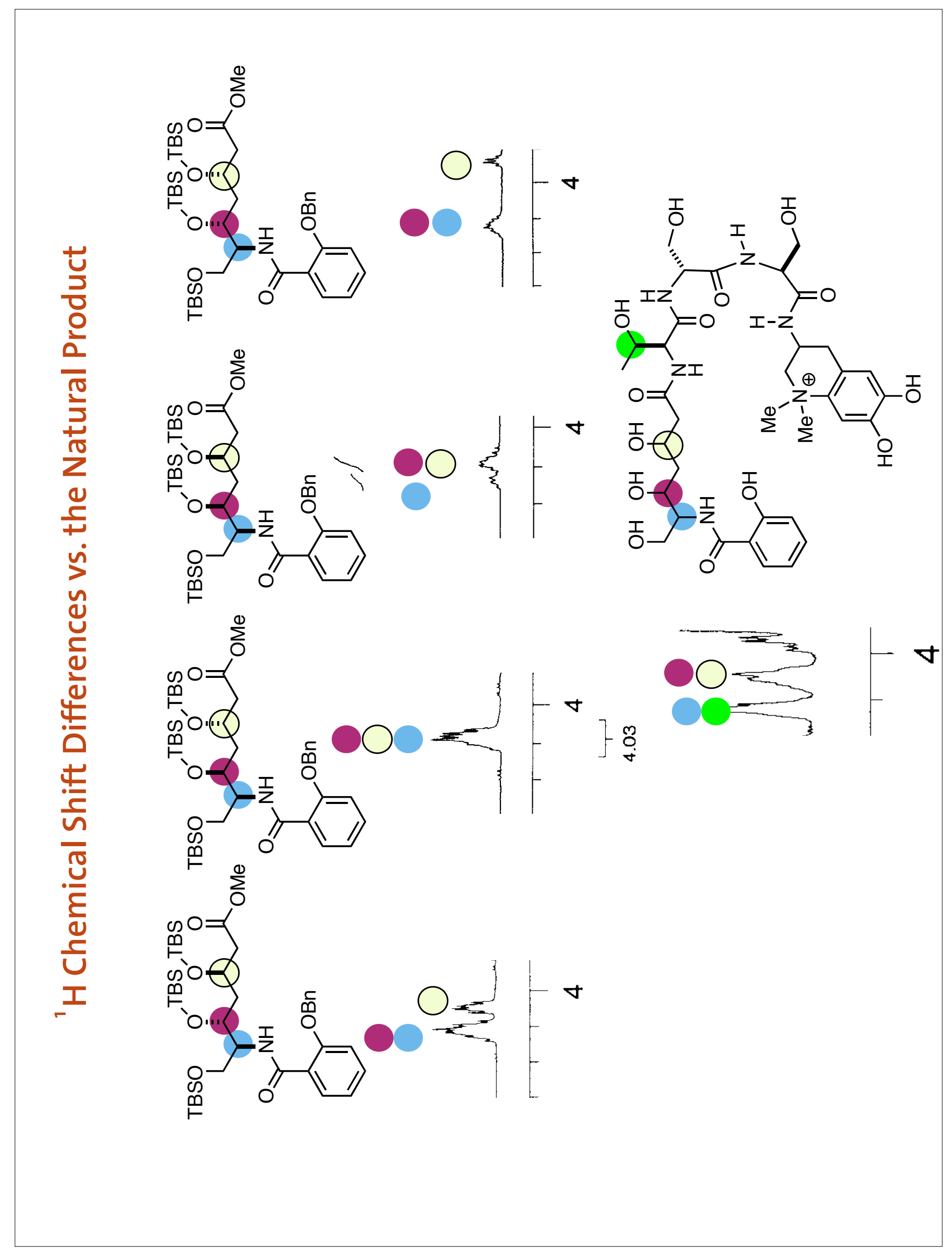




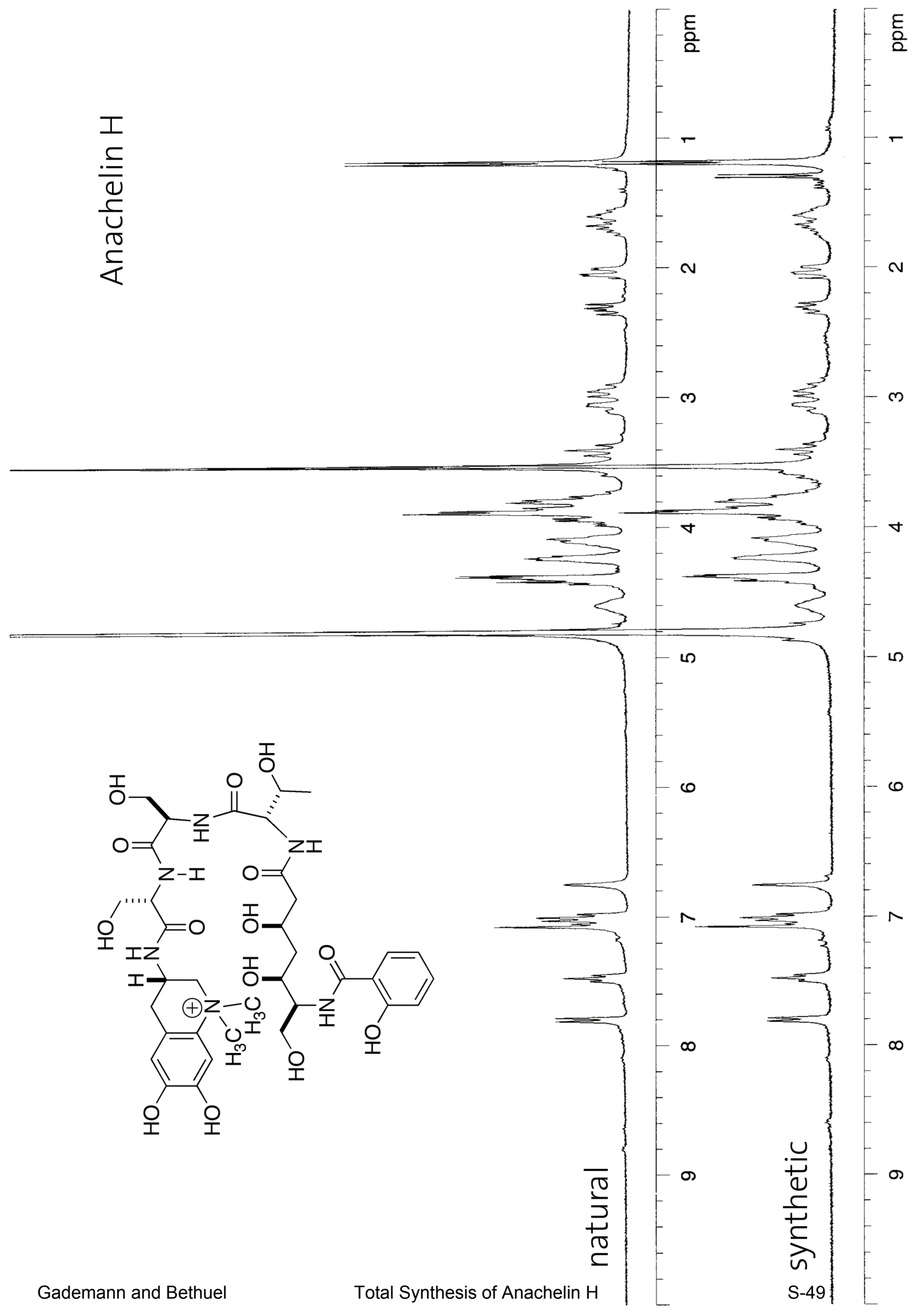




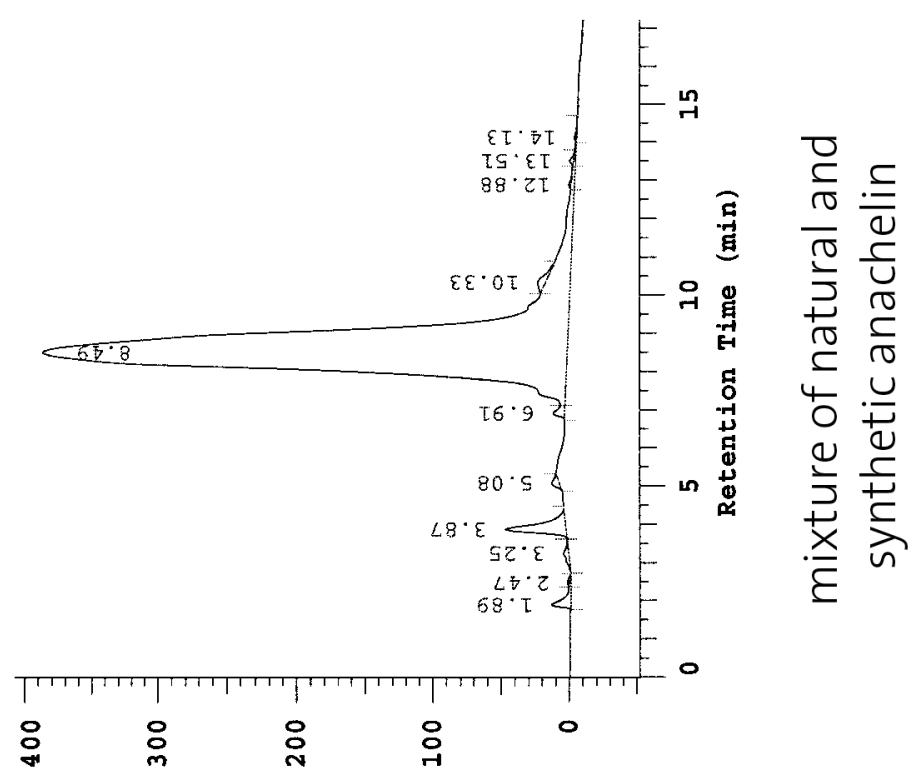

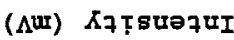

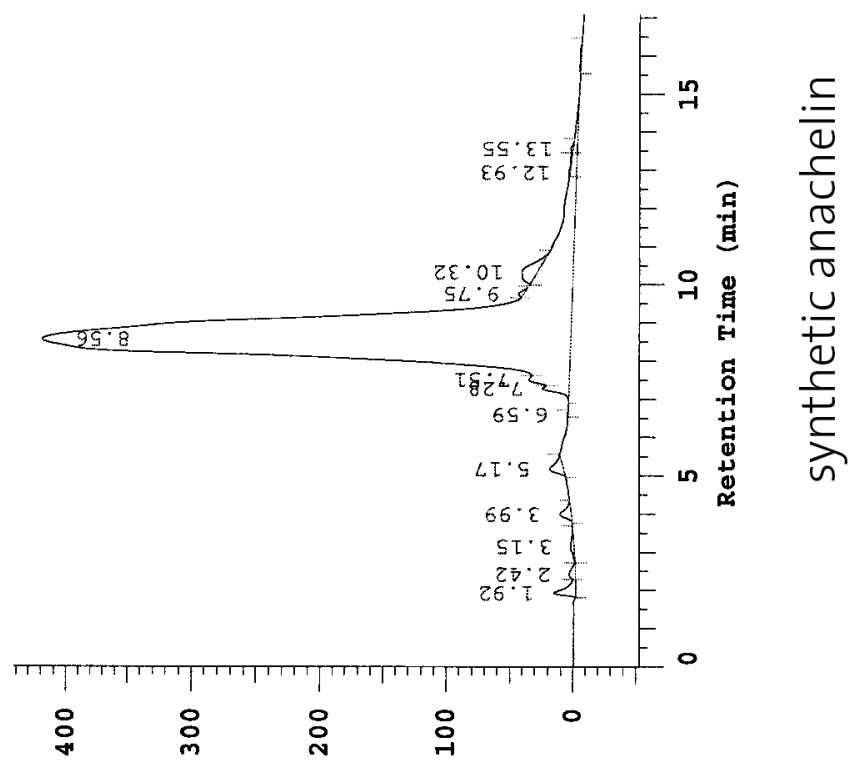

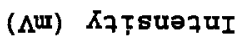

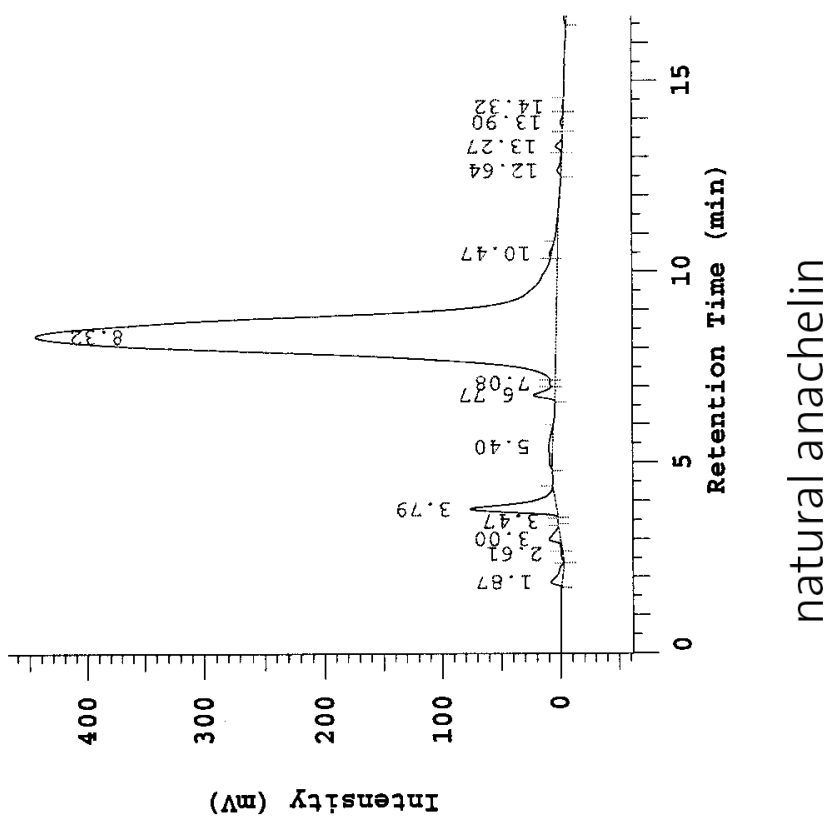

